EPHOU-99-0015

November, 1999

\title{
4-dimensional BF Gravity on the Lattice
}

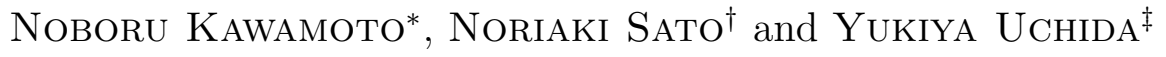 \\ Department of Physics, Hokkaido University \\ Sapporo, 060-0810, Japan
}

\begin{abstract}
We propose the lattice version of $B F$ gravity action whose partition function leads to the product of a particular form of $15-j$ symbol which corresponds to a 4 -simplex. The action is explicitly constructed by lattice $B$ field defined on triangles and link variables defined on dual links and is shown to be invariant under lattice local Lorentz transformation and Kalb-Ramond gauge transformation. We explicitly show that the partition function is Pachner move invariant and thus topological. The action includes the vanishing holonomy constraint which can be interpreted as a gauge fixing condition. This formulation of lattice $B F$ theory can be generalized into arbitrary dimensions.
\end{abstract}

PACS code : 04.60.Nc, 11.15.Ha

Keywords : Lattice gravity, BF theory, Quantum gravity

\footnotetext{
*kawamoto@particle.sci.hokudai.ac.jp

${ }^{\dagger}$ noriaki@particle.sci.hokudai.ac.jp

${ }^{\ddagger}$ uyukiya@particle.sci.hokudai.ac.jp
} 


\section{Introduction}

In the mid 70's nobody expected that it was possible to evaluate the hadron masses by any means in the near future. The later developments of lattice QCD made it possible and gave us an idea that non-perturbative formulation of gauge theory on the lattice is crucial for the realistic numerical analyses of the phenomena based on the field theory like QCD. As for the lepton and quark masses we don't have any possible formulation at this moment. It would, however, be not unreasonable to expect that gravity theory might play a crucial role in the realistic unified model leading to the lepton and quark spectrum calculations. On the other hand it is quite natural to expect that some means to evaluate phenomenological numbers numerically is obviously needed. We believe that a lattice theory will play again a crucial role in the formulation. This line of thinking naturally encourages us to find a formulation of gravity theory on the lattice.

In 2-dimensional gravity the dynamical triangulation of lattice theory was very successful analytically [1] [2] and numerically [3] [4] to evaluate the fractal dimensions [5]. The matrix model for the dynamical triangulation of random surface and the Liouville theory are the analytic means on one hand while the recursive sampling

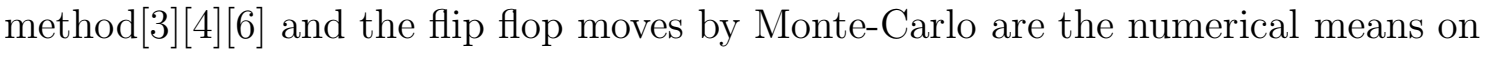
the other in 2-dimensional quantum gravity. In general there is no local action to describe the 2-dimensional quantum gravity.

In 3 dimensions Einstein gravity has been successfully formulated by ChernSimons action as a gauge theory of $\operatorname{ISO}(2,1)$ group[7]. After Turaev and Viro [9] proposed the possibility based on the analyses of $q$-deformed version of PonzanoRegge model that the Ponzano-Regge 8 model formulated by $6-j$ symbols may be the lattice version of Chern-Simons gravity, there are several authors to try to prove the connection of these two formulations 10 11]. The proofs of the connection has been, however, indirect. In our recent paper we have shown by explicit calculations that the partition function of the lattice version of Chern-Simons action indeed leads to the Ponzano-Regge model[12].

In 4 dimensions Ooguri pointed out the possibility that the partition function of $B F$ theory [13] leads to the partition function constructed from $15-j$ symbols 14. This is quite parallel to the 3-dimensional case where the Chern-Simons gravity theory is associated with the Ponzano-Regge model. This 4-dimensional proposal trig- 
gered a new way of defining 4-dimensional quantum gravity theory by spin foam 15 and a new type of 4-dimensional topological invariant by the quantum $q$-deformed formulation[16] [17].

There are plausible arguments that the partition function of the continuum $B F$ theory and 15- $j$ symbols are related, but there is no explicit derivation to connect the two models. Here in this paper we first introduce the lattice version of $B F$ theory and explicitly show that the partition function of the lattice $B F$ theory leads to the product of $15-j$ symbols. We then show explicitly that the particular combination of $15-j$ product is Pachner move invariant and thus the partition function is independent how the 4-dimensional simplicial manifold is divided by the Pachner moves and is thus topological. Then the continuum limit of the lattice $B F$ theory can be taken analytically and leads to the continuum $B F$ action since the partition function is division independent of the 4-dimensional simplicial manifold.

In formulating lattice gravity theory, we intend to couple the formulation of the lattice QCD and Regge calculus 18. It has already been proposed that the link variable of lattice QCD can be used to generate the curvature on the simplexes where Regge calculus claims as the location of gravitational curvature [19] [20].

It is known that the $B F$ theory is not equivalent to the Einstein gravity but if the 2 -form $B$ field is related to the vierbein as $B=*(e \wedge e)$, then the $B F$ action leads to the Einstein-Hilbert action 21. It is also known that the metric can be written by $B$ field directly if some constraints are fulfilled. In this case the Einstein gravity will be formulated by 2 -form field $B$ and equations of motion. In this sense the $B F$ theory is closely related to the gravity theory but in the limited sense. The lattice version of $B F$ theory is, therefore, a very good starting point to the realistic 4-dimensional lattice gravity theory.

One of the authors (N.K.) and Watabiki discovered the formulation to generalize the standard Chern-Simons action into arbitrary dimensions by employing all degrees of form as gauge fields and parameters 22]. It was shown that the topological gravity and the topological conformal gravity can be formulated by 2- and 4-dimensional generalized Chern-Simons action 23]. We show concrete expressions of the generalized Chern-Simons actions in 2, 3 and 4 dimensions where we omit fermionic degrees for simplicity,

$$
S_{2}=-\int \operatorname{Tr}\left\{\phi\left(d \omega+\omega^{2}\right)+\phi^{2} B\right\}
$$




$$
\begin{aligned}
& S_{3}=-\int \operatorname{Str}\left\{\frac{1}{2} \omega d \omega+\frac{1}{3} \omega^{3}-\phi(d B+[\omega, B])+\phi^{2} \Omega\right\}, \\
& S_{4}=-\int \operatorname{Tr}\left\{B\left(d \omega+\omega^{2}\right)+\phi(d \Omega+\{\omega, \Omega\})+\phi B^{2}+\phi^{2} H\right\},
\end{aligned}
$$

which are invariant under the following gauge transformations:

$$
\begin{aligned}
\delta \phi & =[\phi, v], \\
\delta \omega & =d v+[\omega, v]-\{\phi, u\}, \\
\delta B & =d u+\{\omega, u\}+[B, v]+[\phi, b], \\
\delta \Omega & =d b+[\omega, b]+[\Omega, v]-\{B, u\}+\{\phi, U\}, \\
\delta H & =-d U-\{\omega, U\}+\{\Omega, u\}+[H, v]+[B, b]+[\phi, V],
\end{aligned}
$$

where [, ] and $\{$,$\} are, respectively, commutator and anti-commutator, and \phi, \omega$, $B, \Omega, H$ and $v, u, b, U, V$ are, respectively, 0-, 1-, 2-, 3- and 4-form gauge fields and parameters. Here odd forms of gauge fields and even forms of gauge parameters are ordinary even Lie algebra valued fields while the rest of gauge fields and parameters are odd super Lie algebra valued fields 22]. Since all these generalized Chern-Simons actions are formulated by form degrees, the fields of forms are very naturally accommodated on the simplicial manifold.

It is important for us to recognize that 3-dimensional Chern-Simons action and 4-dimensional BF action are the leading terms of the above generalized ChernSimons actions of $S_{3}$ and $S_{4}$. In the previous paper and present paper we focus on the formulation of the leading terms of the 3- and 4-dimensional generalized ChernSimons action as the lattice gravity theory on a simplicial manifold. It is very natural to generalize the lattice formulation of this paper to include all the form degrees of the generalized Chern-Simons action on the lattice. We believe that the matter field will be accommodated via this generalized gauge theory formulation which might lead to the unified theory including gravity on the simplicial manifold 24]. In this sense the $B F$ action on the lattice is the good starting formulation for the generalized gauge theory as well.

In section 2 we first summarize the continuum formulation of $B F$ theory then introduce a lattice version of $B F$ theory. Since the lattice $B F$ theory is a gauge theory, we discuss the gauge invariance of the theory on the lattice in section 3 . We then integrate out the $B$ fields and the link variables and then explicitly show that the partition function of the lattice $B F$ action leads to the product of $15-j$ symbols 
in section 4. We then prove the Pachner move invariance of our partition function by graphical method explicitly in section 5 . We summarize the result with several discussions in the final section.

\section{Formulation of the lattice $B F$ theory}

\subsection{Continuum $B F$ Theory}

We first summarize characteristics of the continuum 4-dimensional $B F$ theory [13]. The action of $B F$ theory is given by

$$
S_{B F}=\int_{M}\langle B, F\rangle
$$

where $M$ is 4-dimensional manifold. We take our gauge group any Lie group $G$ whose Lie algebra $\mathcal{L}_{\mathcal{G}}$ is equipped with an invariant nondegenerate bilinear form $\langle$,$\rangle for the pair B$ and $F$. Here $F$ is curvature 2-form constructed from Lie algebra $\mathcal{L}_{\mathcal{G}}$ valued 1-form $\omega$, and $B$ is dual Lie algebra $\mathcal{L}_{\mathcal{G}}^{*}\left(=\mathcal{L}_{\mathcal{G}}\right.$ in the present case $)$ valued generic 2-form.

It has been noticed since quite sometime that the $B F$ action leads to the Palatini type of Einstein-Hilbert action if we take the Lie group $G$ to be local Lorentz group and identify $B=*(e \wedge e)$ with $*$ as Hodge dual operation to the local Lorentz suffices. In particular the self dual component of the 2 -form $B$ plays a crucial role in formulating the Einstein gravity by the $S L(2, C)$ spinor representation 21.

Using the fact that the 4-dimensional Euclidean version of local Lorentz group is a direct product of chiral $S U(2)$ groups: $S O(4) \simeq S U(2)_{L} \times S U(2)_{R}$, we can factorize the $B F$ action with local Lorentz group into the chiral parts,

$$
S_{B F}=\int_{M} B_{a^{\prime} b^{\prime}} F^{a^{\prime} b^{\prime}}=\int_{M} B_{a}^{+} F_{a}^{+}+\int_{M} B_{a}^{-} F_{a}^{-},
$$

where $F_{a}^{ \pm} \equiv \pm F_{0 a}^{ \pm}, F_{a^{\prime} b^{\prime}}^{ \pm} \equiv F_{a^{\prime} b^{\prime}} \pm \frac{1}{2} \epsilon_{a^{\prime} b^{\prime} c^{\prime} d^{\prime}} F^{c^{\prime} d^{\prime}}$, and similar for $B_{a}^{ \pm}$and $B_{a^{\prime} b^{\prime}}^{ \pm}$. The suffices, $a^{\prime}, b^{\prime}, \ldots$ are local Lorentz suffices while $a$ is the chiral $S U(2)$ suffix. In this paper we only consider the one chiral, say left handed, counterpart of the action. We may consider that the right-handed part is nothing but the copy of the left-handed part, at least in the continuum limit. On the lattice there would be a possibility to accommodate the same copy of chiral counterpart which we will discuss later. If we take the left-chiral part of the action, we can formulate the lattice $S U(2) B F$ 
theory by naive extension from 3-dimensional lattice Chern-Simons gravity into 4 dimensions.

In formulating left-chiral $B F$ model, we can expand both $B$ and $F$ by the Pauli matrices, $\sigma^{a}(a=1,2,3)$ since $S U(2)$ has self-dual Lie algebra,

$$
B=\frac{1}{2} B^{a} \sigma^{a}, \quad F=\frac{1}{2} F^{a} \sigma^{a} .
$$

Hereafter we omit the suffix + to denote the left chirality. Then the action of $S U(2)$ $B F$ theory leads to

$$
S_{B F}=\int_{M} \operatorname{tr} B F=\frac{1}{2} \int_{M} B^{a} F^{a},
$$

where the trace is taken for the Pauli matrices. It is worth to mention that the factor 2 difference between the chiral part of $B F$ action in (2.2) and the above action (2.4) is due to the fact that the chiral decomposition of the fundamental representation for $S O(4)$ generator is reducible into the direct sum of two Pauli matrices.

There are two independent gauge transformations in this theory. One of them is the local Lorentz transformation,

$$
\delta_{\tau} \omega^{a}=D \tau^{a}, \quad \delta_{\tau} B^{a}=[B, \tau]^{a},
$$

where $\tau$ is 0 -form gauge parameter. There is another independent gauge transformation

$$
\delta_{u} \omega^{a}=0, \quad \delta_{u} B^{a}=D u^{a}
$$

where $u^{a}$ is 1 -form gauge parameter according to the 2 -form nature of $B^{a}$. This transformation may be called Kalb-Ramond symmetry transformation 25] and corresponds to the diffeomorphism transformation of dreibein $e$ in 3 dimensions. This transformation is on-shell reducible. In fact the following gauge change of the gauge parameter $u$ itself by 0 -form gauge parameter $v^{a}$,

$$
\delta_{v} u^{a}=D v^{a}
$$

leads to the vanishing contribution of the gauge change

$$
\delta_{v}\left(\delta_{u} B^{a}\right)=D \delta_{v} u^{a}=D D v^{a}=[F, v]^{a}=0,
$$

where we have used the equation of motion $F=0$. 


\subsection{Lattice $B F$ Gravity Action}

We intend to formulate a gravity version of lattice gauge theory where the product of link variables $U$ along the boundary of a square plaquette leads to the exponential of curvature. Thus we may identify the curvature locating at the center of the plaquette and then the trace leads to the Yang-Mills action in the leading order of the lattice constant in the lattice QCD. On the other hand Regge calculus tells us that the curvature is located at sites in 2 dimensions, links in 3 dimensions, triangles in 4 dimensions and so on. It is thus very natural to formulate the gravity version of lattice gauge theory in terms of the link variable $U$ and take a product of $U$ variables surrounding the simplexes suggested by Regge calculus [19] [20].

In fact in 3 dimensions, we have successfully formulated lattice Chern-Simons gravity based on the correspondence between the lattice gauge theory and Regge calculus[12]. The link variable $U(\tilde{l})=e^{\omega(\tilde{l})}$ is defined on the dual link $\tilde{l}$ which is located on the boundary of dual plaquette $\tilde{P}$. Since the dual of the dual plaquette $\tilde{P}$ is original link $l$ which intersect $\tilde{P}$ in 3 dimensions, the curvature is located in the center of the dual plaquette or equivalently on the original link and thus consistent with the Regge calculus. We needed to introduce vanishing holonomy constraint which enforces the parallelism of the dreibein $e^{a}$ and the lattice curvature $F^{a} \equiv \frac{1}{2} \epsilon^{a b c} F^{b c}$ to obtain gravity theory. It turns out that the partition function formulated in this way exactly coincides with that of the Ponzano-Regge model. Then the action leads to the continuum Chern-Simons gravity since the PonzanoRegge partition function is Alexander move invariant and thus invariant under the division of the simplicial manifold and then the naive continuum limit can be taken.

In this paper we extend the formulation of 3-dimensional lattice gravity into 4 dimensions. We formulate a lattice version of $S U(2) B F$ theory. We consider a piecewise linear 4-dimensional simplicial manifold. According to the Regge calculus, curvature is located at a triangle $t$. There are in general several 4-simplexes which have the triangle $t$ in common. A dual link $\tilde{l}$ is defined as the line connecting the center of neighbouring 4-simplexes and is located on the boundary of the dual plaquette $\tilde{P}$. We introduce dual link variables $U(\tilde{l})=e^{i \omega(\tilde{l})}$ on the dual link $\tilde{l}$ 国. Then the product of the dual link variables $U(\tilde{l})$ along the boundary of the dual plaquette

\footnotetext{
${ }^{\S}$ In [12] we have used anti-hermitian generators of $S O(3)$ and thus denoted the link variable as $U=e^{\omega}$ while here we use hermitian generators of $S U(2)$ and thus denote the link variable as $U=e^{i \omega}$.
} 
$\tilde{P}$ leads to a curvature located on the center of the dual plaquette $\tilde{P}$ which coincides with the center of the triangle $t$ in 4 dimensions. We then locate the 2 -form field $B$ on the original triangle $t$. See Fig.[1.

We define the "curvature" $F(t)$ located on the triangle $t$ by the following equation:

$$
\prod_{\tilde{l} \in \partial \tilde{P}} U(\tilde{l}) \equiv e^{i F(t)} .
$$

The leading term in $F$ with respect to the lattice unit is the ordinary curvature $d \omega+\omega^{2}$ similar in structure to the ordinary lattice gauge theory, but here we define the lattice "curvature" $F$ in such a way that it contains all orders of terms of lattice unit.

Using these variables, we consider the following lattice version of the $B F$ action:

$$
S_{L B F}=\sum_{t} \operatorname{tr}\left(-i B(t)\left[\ln \prod_{\tilde{l} \in \partial \tilde{P}} U(\tilde{l})\right]\right)=\frac{1}{2} \sum_{t} B^{a}(t) F^{a}(t) .
$$

In formulating continuum Chern-Simons gravity in 3 dimensions, we need to impose a torsion free condition as an equation of motion at the classical level. The torsion free nature is lost at the quantum level when we integrate out the dreibein and spin connection. In formulating lattice Chern-Simons gravity and showing the equivalence with the Ponzano-Regge model in 3 dimensions, we needed to introduce the following vanishing holonomy constraint which relates the dreibein and spin connection even at the quantum level:

$$
\left[\mathbb{\mathbb { Q }}_{\tilde{l} \in \partial \tilde{P}} U(\tilde{l})\right]^{a b} e^{b}=e^{a},
$$

where $a, b$ are $S O(3)$ suffices. This constraint has been interpreted as a gauge fixing condition of lattice gauge diffeomorphism transformation. Geometrically the con-

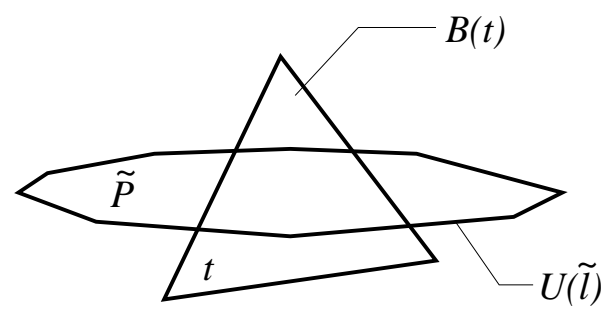

Figure 1: $\tilde{P}$ is the dual plaquette dual to the triangle $t$ associated to the 2 -form $B(t)$. Dual link variable $U=e^{i \omega}$ is located on the dual link which constructs the boundary of the dual plaquette $\tilde{P}$. 
straint is equivalent to the parallelism of the dreibein and the curvature: $e^{a} \propto F^{a}$, where $F^{a} \equiv \frac{1}{2} \epsilon^{a b c} F^{b c}$.

In contrast with the 3 -dimensional case we introduce the following constraint in 4 dimensions

$$
\left[\left(\prod_{\tilde{l} \in \partial \tilde{P}} U(\tilde{l})\right) B\left(\prod_{\tilde{l} \in \partial \tilde{P}} U(\tilde{l})\right)^{\dagger}\right]^{\alpha \beta}=B^{\alpha \beta},
$$

where $\alpha \beta$ are suffices of $S U(2)$ spinor representation. Geometrically the constraint means that the each of spinor suffix of $B^{\alpha \beta}$ field located at the center of a triangle can be parallel transported in the opposite direction along the boundary of the dual plaquette $\tilde{P}$ and comes back to the starting point and should coincide with the original direction and thus the holonomy of each suffix vanishes. We now derive more concrete expression for the constraint (2.12) which is equivalent to the following relations:

$$
\sum_{n=0}^{\infty} \frac{i^{n}}{n !}[F, \cdots[F,[F, B]] \cdots]=B .
$$

This equation can be satisfied if

$$
[F, B]=i \epsilon_{a b c} F^{a} B^{b} \frac{\sigma^{c}}{2}=0
$$

or equivalently $B^{a} \propto F^{a}$.

Analogous to the arguments in 3 dimensions, the constraint thus leads to the parallelism of the 2-form $B^{a}$ and the curvature: $B^{a} \propto F^{a}$. More precisely the constraint can be rewritten in a concrete form by taking into account the parallel and antiparallel nature of $B^{a}$ and $F^{a}$

$$
\frac{B^{3}}{|B|}\left[\prod_{a=1}^{2} \delta\left(\frac{F^{a}}{|F|}+\frac{B^{a}}{|B|}\right)+\prod_{a=1}^{2} \delta\left(\frac{F^{a}}{|F|}-\frac{B^{a}}{|B|}\right)\right],
$$

where $|B| \equiv \sqrt{B^{a} B^{a}}$ and $|F| \equiv \sqrt{F^{a} F^{a}}$ are the length of $B^{a}$ and $F^{a}$, respectively. The term $B^{3} /|B|$ is needed to keep the rotational invariance of the expression.

One of the important characteristic of the lattice $B F$ action (2.10) is that the discreteness of the length of the 2-form field $|B|$ comes out as a natural consequence of the specific choice of the logarithmic action. We first note the following identity:

$$
e^{i|F| I}=\cos \left(\frac{|F|}{2}\right)+i 2 I \sin \left(\frac{|F|}{2}\right),
$$

where $I=\frac{F^{a}}{|F|} \frac{\sigma^{a}}{2}$ with $\sigma^{a}$ as Pauli matrix, then

$$
e^{i 4 \pi n I}=1, \quad n \in \mathrm{Z} .
$$


Using the above relation and $F^{a} \propto B^{a}$ by the constraint (2.12), we find that our lattice $B F$ action $S_{L B F}$ has the following ambiguity:

$$
\begin{aligned}
S_{L B F} & =\sum_{t} \operatorname{tr}\left(-i B(t) \ln e^{F(t)}\right) \\
& =\sum_{t} \operatorname{tr}\left(-i B(t) \ln e^{F(t)+i 4 \pi n I}\right) \\
& =S_{L B F}+\frac{1}{2} \sum_{t} 4 \pi n|B(t)|
\end{aligned}
$$

This ambiguity leads to an ambiguity in the partition function

$$
Z=\int \mathcal{D} U \mathcal{D} B e^{i S_{L B F}}=\int \mathcal{D} U \mathcal{D} B e^{i S_{L B F}+i \sum_{t} 2 \pi n|B|} .
$$

Imposing the single valuedness of $e^{i S_{L B F}}$, we obtain the constraint that $\sum_{t}|B(t)|$ should be integer, or equivalently $|B(t)|$ should be integer. We can now write down the explicit form of partition function with constraints

$$
Z=\int \mathcal{D} U \mathcal{D} B \delta\left((\mathbb{\mathbb { }} U(\tilde{l})) B(\mathbb{\mathbb { }} U(\tilde{l}))^{\dagger}-B\right) \sum_{N} \delta(|B|-N) e^{i S_{L B F}}
$$

where $N \in Z$.

It is important to notice that the length of the 2 -form $|B(t)|$ is proportional to the area of triangle $t$ on which the 2 -form field $B^{a}(t)$ is defined. It is then interesting to note that the "area of the triangle" is discretized due to the logarithmic form of our lattice $B F$ action. It has already been pointed out by Rovelli and Smolin that the area of triangle in 4-dimensional gravity is discretized in the square root of angular

momentum square $\sqrt{J(J+1)}$ by investigating an area operator 26. The origin of the discreteness of the area of triangle in our formulation is quite different from that of Rovelli and Smolin.

\section{Gauge Invariance on the Lattice}

The gauge transformations of the continuum $B F$ theory have been given by the local Lorentz transformation (2.5) and the Kalb-Ramond transformation (2.6). Under the local Lorentz transformation both of the $B$ field and curvature $F$ transform adjointly in the continuum theory

$$
\delta_{\tau} B^{a}=[B, \tau]^{a}, \quad \delta_{\tau} F^{a}=[F, \tau]^{a} .
$$


We consider that the lattice versions of the local Lorentz gauge parameters are located on the dual sites and the center of the original triangles, the same point where the $B$ field is located. Then the dual link variable $U(\tilde{l})=e^{i \omega(\tilde{l})}$ transforms under the lattice local Lorentz transformation as

$$
U(\tilde{l}) \rightarrow V^{-1} U(\tilde{l}) V^{\prime}
$$

where $V=\exp \left(\frac{i}{2} \tau^{a} \sigma^{a}\right)$ is the $S U(2)$ matrix on the dual site and $V^{\prime}$ is the one on the neighboring site. Then the following lattice counterpart of the local Lorentz transformation:

$$
\begin{aligned}
& B(t) \rightarrow V^{-1} B(t) V, \\
& F(t) \rightarrow V^{-1} F(t) V,
\end{aligned}
$$

leads to the obvious invariance of the action (2.4).

There are, however, some subtleties on the gauge invariance of the lattice action due to the fact that the center of the triangle where $B$ field is located and the dual links where dual link variables $U(\tilde{l})$ are located are not connected by a link variable $U$. We can arbitrarily choose a dual site and introduce new link variable to bridge between the dual site and the center of the original triangle to identify the lattice local Lorentz transformation. The newly defined link variables, however, can be gauged away. The details of these arguments go parallel to the 3-dimensional Chern-Simons gravity case and can be found in [12].

Next we will investigate the lattice version of Kalb-Ramond transformation. Since the invariance nature of the continuum action under this transformation is due to the Bianchi identity,

$$
D F=d F+[\omega, F]=0
$$

we need to identify Bianchi identity on the lattice. We will consider the integrated version of the Bianchi identity,

$$
\int_{M} D F=\int_{\partial M} F+\int_{M}[\omega, F]=0
$$

where $M$ is a 3 -dimensional manifold corresponding to the 3 -form nature of $D F$ in (3.4).

In 3 dimensions the geometrical structure of the manifold $M$ can be visually well understood since the dual cell of a original site associated to the 0-form gauge parameter of the gauge diffeomorphism transformation is easily recognized. This 


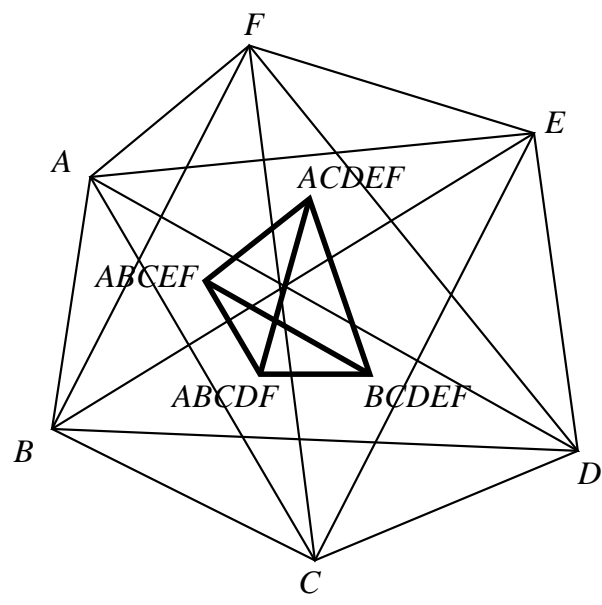

Figure 2: A simple setup to show the integrated lattice Bianchi identity. $A, B, C$, $D, E$ and $F$ are sites and $A C D E F, A B C E F, A B C D F, B C D E F$ are the centers of 4 -simplex. Thick lines are the dual links and thin lines are original links.

type of setup was just what we needed to show the 3-dimensional lattice counterpart of the Bianchi identity [12].

Correspondingly in 4 dimensions, we have to consider the dual cell of the original link $C F$ as a 3 -dimensional simplicial submanifold $M$ since the gauge parameter of the Kalb-Ramond transformation (2.6), which we now identify as the parameter on the link $C F$, is 1 -form in 4 dimensions. In general the 3-dimensional cell dual to a given link may have complicated simplicial structure. For simplicity we here consider the dual cell as a tetrahedron. The tetrahedron as the dual cell has four dual sites which correspond to the center of original 4-simplexes. We then need to introduce four original sites $A, B, D$ and $E$ to obtain four 4-simplexes which share the common link $C F$ as shown in Fig.2. With this setup we can discuss the invariance of the lattice $B F$ action (2.10) under a lattice version of Kalb-Ramond transformation.

We introduce the following notations: $U_{1} \equiv U_{A C D E F \rightarrow A B C D F}$ is a dual link variable on the dual link which connect the dual sites or equivalently the center of original links denoted as $A C D E F$ and $A B C D F$. The 2-form gauge field $B_{1} \equiv \int_{B C F} B_{\mu \nu} d x^{\mu} d x^{\nu}$ is located on the center of the triangle $B C F$. The 1-form gauge parameter $u_{0} \equiv \int_{F C} u_{\mu} d x^{\mu}$ is located on the original link $F C$. The 0 -form gauge parameter $v_{1} \equiv v(B)$ is located on the original site $B$. Here we summarize 
the notations:

$$
\begin{aligned}
& \left\{\begin{array}{c}
U_{1} \equiv U_{A B C D F \rightarrow A C D E F}, U_{2} \equiv U_{A B C D F \rightarrow B C D E F}, U_{3} \equiv U_{A B C D F \rightarrow A B C E F}, \\
U_{4} \equiv U_{B C D E F \rightarrow A B C E F}, U_{5} \equiv U_{A B C E F \rightarrow A C D E F}, U_{6} \equiv U_{A C D E F \rightarrow B C D E F},
\end{array}\right. \\
& \left\{\begin{array}{l}
B_{1} \equiv \int_{B C F} B_{\mu \nu} d x^{\mu} d x^{\nu}, B_{2} \equiv \int_{A C F} B_{\mu \nu} d x^{\mu} d x^{\nu} \\
B_{3} \equiv \int_{C D F} B_{\mu \nu} d x^{\mu} d x^{\nu}, B_{4} \equiv \int_{C E F} B_{\mu \nu} d x^{\mu} d x^{\nu}
\end{array}\right. \\
& \left\{\begin{array}{l}
u_{0} \equiv \int_{F C} u_{\mu} d x^{\mu}, u_{1} \equiv \int_{C B} u_{\mu} d x^{\mu}, u_{2} \equiv \int_{B F} u_{\mu} d x^{\mu}, u_{3} \equiv \int_{C A} u_{\mu} d x^{\mu}, u_{4} \equiv \int_{A F} u_{\mu} d x^{\mu}, \\
u_{5} \equiv \int_{C D} u_{\mu} d x^{\mu}, u_{6} \equiv \int_{D F} u_{\mu} d x^{\mu}, u_{7} \equiv \int_{C E} u_{\mu} d x^{\mu}, u_{8} \equiv \int_{E F} u_{\mu} d x^{\mu},
\end{array}\right. \\
& \left\{v_{1} \equiv v(B), v_{2} \equiv v(A), v_{3} \equiv v(D), v_{4} \equiv v(E), v_{5} \equiv v(F), v_{6} \equiv v(C)\right. \text {. }
\end{aligned}
$$

We first note the following identity,

$$
\prod U \equiv U_{2} U_{4} U_{3}^{-1} \cdot U_{3} U_{5} U_{1}^{-1} \cdot U_{1} U_{6} U_{2}^{-1} \cdot U_{2} U_{6}^{-1} U_{5}^{-1} U_{4}^{-1} U_{2}^{-1}=1
$$

Using the following definitions of curvature on the triangles,

$$
e^{F_{1}} \equiv U_{2} U_{4} U_{3}^{-1}, e^{F_{2}} \equiv U_{3} U_{5} U_{1}^{-1}, e^{F_{3}} \equiv U_{1} U_{6} U_{2}^{-1}, e^{F_{4}} \equiv U_{2} U_{6}^{-1} U_{5}^{-1} U_{4}^{-1} U_{2}^{-1}
$$

and the Baker-Hausdorff formula,

$$
e^{A} \cdot e^{B}=\exp \left(A+B+\frac{1}{2}[A, B] \cdots\right)
$$

we can rewrite the above identity in the following form:

$$
0=\ln \prod U=\ln \left(e^{F_{1}} \cdot e^{F_{2}} \cdot e^{F_{3}} \cdot e^{F_{4}}\right)=\sum_{k=1}^{4} F_{k}+\sum_{k=1}^{4}\left[\Omega_{k}, F_{k}\right] .
$$

Here we have introduced the following variables $\Omega_{k}$,

$$
\begin{array}{ll}
\Omega_{1}=-\frac{1}{4}\left(F_{2}+F_{3}+F_{4}\right)+\cdots, & \Omega_{2}=-\frac{1}{4}\left(-F_{1}+F_{3}+F_{4}\right)+\cdots, \\
\Omega_{3}=-\frac{1}{4}\left(-F_{1}-F_{2}+F_{4}\right)+\cdots, & \Omega_{4}=-\frac{1}{4}\left(-F_{1}-F_{2}-F_{3}\right)+\cdots,
\end{array}
$$

which can be evaluated order by order of the curvature $F$ by using the BakerHausdorff formula. We identify (3.9) as the lattice version of the Bianchi identity (3.4).

Now we consider the lattice version of the gauge transformation for the 2-form gauge field $B_{k}$. We first note that $B_{1}$ field is located on the center of the triangle 
$B C F$ and thus the gauge transformation of $B_{1}$ field is associated with the gauge parameters $u_{0}, u_{1}$ and $u_{2}$ located on the boundary $F C, C B$ and $B F$, respectively. Then the lattice version of $B_{k}$ transformation will be given as follows:

$$
\delta B_{k}=u_{0}+u_{2 k-1}+u_{2 k}-\left[\Omega_{k}, u_{0}\right]-\left[\Omega_{2 k-1}^{\prime}, u_{2 k-1}\right]-\left[\Omega_{2 k}^{\prime \prime}, u_{2 k}\right]
$$

where $\Omega_{k}$ is defined in (3.10) while $\Omega_{2 k-1}^{\prime}$ and $\Omega_{2 k}^{\prime \prime}$ are the similar quantities associated with the gauge parameters $u_{2 k-1}$ and $u_{2 k}$ defined on the links just as $\Omega_{k}$ is associated with the gauge parameter $u_{0}$ on the link $F C$. Then the transformation of the action leads to

$$
\begin{aligned}
\delta S_{L B F} & =\sum_{k=1}^{4} \operatorname{tr}\left(\delta B_{k} \cdot F_{k}\right)+\cdots \\
& =\sum_{k=1}^{4} \operatorname{tr}\left(\left(u_{0}+u_{2 k-1}+u_{2 k}-\left[\Omega_{k}, u_{0}\right]-\left[\Omega_{2 k-1}^{\prime}, u_{2 k-1}\right]-\left[\Omega_{2 k}^{\prime \prime}, u_{2 k}\right]\right) F_{k}\right)+\cdots \\
& =\operatorname{tr}\left(u_{0} \sum_{k=1}^{4}\left(F_{k}+\left[\Omega_{k}, F_{k}\right]\right)\right)+\cdots \\
& =0
\end{aligned}
$$

where in the last expression we have only picked up the terms associated with $u_{0}$. We can identify this transformation as the Kalb-Ramond transformation on the lattice. Thus the lattice $B F$ action is invariant under the transformation (3.11) due to the lattice version of Bianchi identity (3.9).

In 4 dimensions the Kalb-Ramond transformation is on-shell reducible. We now show how the reducibility of the 2 -form gauge transformation is realized on the lattice. Due to the equation of motion $F=0$, we obtain $\Omega=0$ since $\Omega$ is a polynomial of $F$ as we can see in the definition (3.10). Hence the transformation of $B$ in (3.11) can be reduced as follows:

$$
\delta B_{k}=u_{2 k-1}+u_{2 k}+u_{0}
$$

which corresponds to the continuum gauge transformation (2.6). Considering the continuum reducibility relation, we identify the transformation of 1 -form gauge parameter $u$ defined on a link as the difference of 0 -form gauge parameter defined on a site,

$$
\delta u(A B)=v(B)-v(A)
$$


more explicitly,

$$
\begin{gathered}
\delta u_{2 k}=v_{5}-v_{k}, \\
\delta u_{2 k-1}=v_{k}-v_{6}, \\
\delta u_{0}=v_{6}-v_{5} .
\end{gathered}
$$

Then the continuum reducibility relation (2.8) leads to

$$
\delta\left(\delta B_{k}\right)=\left(v_{k}-v_{6}\right)+\left(v_{5}-v_{k}\right)+\left(v_{6}-v_{5}\right)=0,
$$

on the lattice. We can consider that this result is corresponding to the on-shell reducibility of the Kalb-Ramond transformation on the lattice.

We now point out that the constraint (2.12) or equivalently (2.15) breaks the lattice version of Kalb-Ramond transformation while the lattice $B F$ action itself is invariant under the transformation as shown in the above. The lattice $B$ field is transformed but the lattice curvature is unchanged under the Kalb-Ramond gauge transformation. Therefore we can use a part of degrees of freedom of the lattice 1-form gauge parameter $u_{k}$ to align the 2-form $B^{a}$ field and the curvature $F^{a}$ in accordance with the constraint. In fact the reducibility allows the total gauge degrees of freedom of the 1 -form gauge parameter $u_{\mu}^{a}$ to be $4 \times 3-3=9$, while the parallel or anti-parallel nature of the 2 -form chiral $B_{\mu \nu}^{a}$ and $F_{\mu \nu}^{a}$ needs $6 \times 3 / 2=9$ constraints where we have taken into account the chiral nature for the space-time suffix as well. There are thus necessary degrees of freedom to fulfill the constraint. The 3 degrees of freedom out of 9 should be exhausted to adjust the discrete nature of the $B^{a}$ field. We now claim that the vanishing holonomy constraint can be identified as the "gauge fixing condition" of the Kalb-Ramond gauge transformation on the lattice.

\section{Calculation of the Partition Function}

In our previous paper we have explicitly shown that 3-dimensional version of lattice Chern-Simons action with the constraint of vanishing holonomy along dual links leads to the Ponzano-Regge model formulated by $6-j$ symbol[12]. Analogously to the 3-dimensional case we explicitly show that the lattice version of $B F$ action (2.10) with the vanishing holonomy constraint (2.12) leads to a topological gravity model formulated by $15-j$ symbol in 4 dimensions.

The partition function with the constraint (2.12) or equivalently (2.15) and the 
discreteness of $|B|$ taken into account is given by

$$
\begin{aligned}
Z=\int \mathcal{D} U & \prod_{t} Z_{t} \\
Z_{t}=\int d^{3} B & \frac{B^{3}}{|B|}\left[\prod_{a=1}^{2} \delta\left(\frac{F^{a}}{|F|}+\frac{B^{a}}{|B|}\right)+\prod_{a=1}^{2} \delta\left(\frac{F^{a}}{|F|}-\frac{B^{a}}{|B|}\right)\right] \\
& \times \sum_{J=0}^{\infty} \delta(|B|-2 J) e^{\frac{i}{2} B^{a} F^{a}} \quad\left(J \in \frac{\mathrm{Z}}{2}\right)
\end{aligned}
$$

where $Z_{t}$ is the part of partition function associated with a triangle $t$. We have introduced the discrete nature of $|B|$ by using the following relation:

$$
\int_{\left|B_{i}\right|}^{\left|B_{f}\right|} d|B|=\int_{\left|B_{i}\right|}^{\left|B_{f}\right|} \sum_{J=0}^{\infty} \delta(|B|-2 J) d|B|,
$$

where $J \in \mathrm{Z} / 2$ and thus $\left|B_{f}\right|$ and $\left|B_{i}\right|$ should be integer.

We can now evaluate the $B$ integral of $Z_{t}$ straightforwardly thanks to the delta functions and obtain

$$
Z_{t}=\sum_{J} 8 J^{2} \cos (J|F|)
$$

At this stage it is important to recognize that arbitrary change of the normalization constant for the lattice $B F$ action (2.10) gives the same result for (4.4). This can be understood by the following arguments: Suppose we take the action of the form $S_{L B F}=\frac{\alpha}{2} \sum_{t} B^{a}(t) F^{a}(t)$, we need to take $\alpha|B(t)|=2 J(J \in \mathrm{Z} / 2)$, based on the similar arguments as in section 2. Taking into account the vanishing holonomy constraint (2.12) or equivalently (2.15), the action essentially leads to the form $S_{L B F}=\sum_{t} J|F(t)|$ and thus to (4.4).

Using the following formula for the character $\chi_{J}$ of the spin- $J$ representation of $S U(2)$

$$
\chi_{J}\left(e^{i \theta^{a} J_{a}}\right)=\chi_{J}(|\theta|)=\frac{\sin \left((2 J+1) \frac{|\theta|}{2}\right)}{\sin \left(\frac{|\theta|}{2}\right)},
$$

where $|\theta|$ is the length of $\theta^{a}$, we find

$$
\chi_{J}(|F|)-\chi_{J-1}(|F|)=2 \cos (J|F|) .
$$

Hence we can naively calculate the triangle partition function,

$$
\begin{aligned}
Z_{t} & =\sum_{J=1}^{\infty} 4 J^{2}\left(\chi_{J}-\chi_{J-1}\right) \\
& =-4 \sum_{J=0}^{\infty}(2 J+1) \chi_{J}
\end{aligned}
$$


This calculation is not precise, because the summation is not convergent. We can, however, justify the above calculation by introducing the heat kernel regularization. The details of the regularization procedure can be found in our previous paper 112].

Integrating out $B$ field and introducing heat kernel regularization and dividing the unimportant constant factor, we obtain the total partition function

$$
Z=\int \mathcal{D} U \prod_{t} \sum_{J=0}^{\infty}(2 J+1) \chi_{J}\left(\prod_{\tilde{l} \in \partial \tilde{P}} U(\tilde{l})\right) e^{-J(J+1) \tau}
$$

where a regularization parameter $\tau$ is introduced. The parameter $\tau$ will be sent to zero $(\tau \rightarrow 0)$ at the end of calculation.

It is interesting to note that the formulation has close similarity with the 3dimensional case. In 3 dimensions the angular momentum $J$ is associated to the original link while it is associated to a triangle in 4 dimensions. This has natural correspondence with Regge calculus in the sense that the discretized angular momentum $J$ is localized at the simplexes where the gravitational curvature is localized.

We now carry out $\mathcal{D} U$ integration of this partition function. Thanks to the character of the partition function, $\mathcal{D} U$ integration is straightforward. We first note the following relation specific to the character:

$$
\chi_{J}\left(\prod_{\tilde{l} \in \partial \tilde{P}} U(\tilde{l})\right)=\sum_{\left\{k_{i}\right\}} \prod_{i=1}^{m} D_{k_{i} k_{i+1}}^{J}\left(U_{i}\right) \quad\left(k_{m+1}=k_{1}\right),
$$

where $D_{k_{i} k_{i+1}}^{J}\left(U_{i}\right)$ is the $D$-function, a spin $J$ matrix representation for a $S U(2)$ group element $U_{i} \equiv U\left(\tilde{l}_{i}\right)$ located on the dual link $\tilde{l}_{i}$. Here we assume that there are $m$ dual link variables $U_{i}$ along the boundary of dual plaquette $\tilde{P}$.

We then clear out the geometrical structure of a 4-simplex. A 4-simplex can be constructed by five sites for which we name $A, B, C, D$ and $E$. We may denote the 4-simplex itself composed of these sites as $A B C D E$, which has five tetrahedra $B C D E, A C D E, A B D E, A B C E$ and $A B C D$ on the boundary. Each tetrahedron, such as $B C D E$, has boundary triangles $C D E, B D E, B C E$ and $B C D$ and so on. In this way we can specify all the simplexes included in the 4-simplex $A B C D E$. We can graphically denotes the geometrical structure of the 4-simplex $A B C D E$. See Fig. 3.

We now clarify the locations of the $U_{i}$ and $D$-functions on the 4-simplexes. The center of the figure in Fig. 3 is corresponding to that of the 4-simplex $A B C D E$ which has five neighboring 4-simplexes and is connected to the 4-simplexes by five dual 


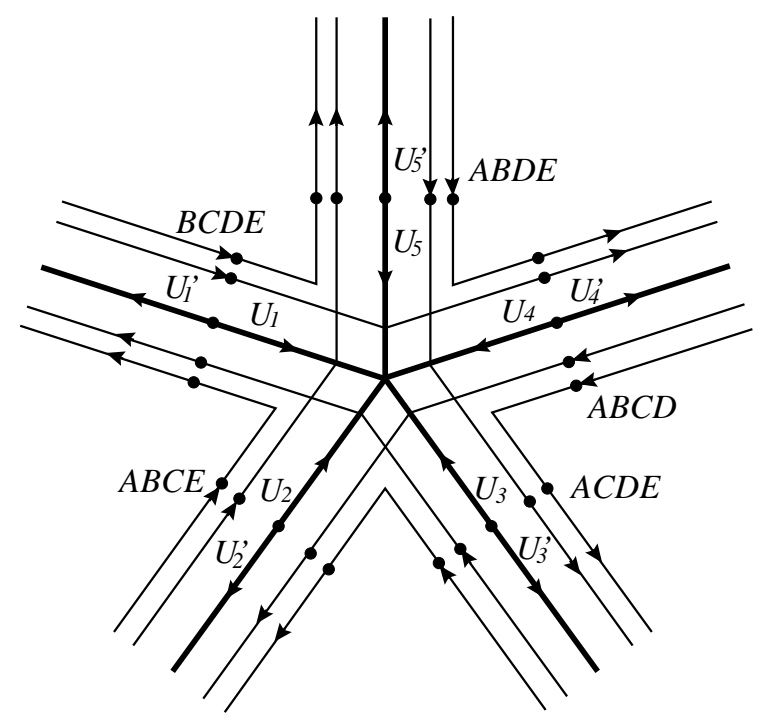

Figure 3: Graphical presentation of geometrical structure of a 4-simplex $A B C D E$

links denoted by the thick lines. The five points on the five thick lines correspond to the center of the five boundary tetrahedra, $B C D E, A C D E, A B D E, A B C E$ and $A B C D$, respectively. Arrows on the dual links indicate the direction of the dual link variables $U_{i}, U_{i}^{\prime}$ and the thin lines with arrows indicate the direction of the product of the $D$-functions. As we can see in (4.8) the product of the $D$-functions goes around a original triangle associated with the representation $J$. For example the boundary triangle between the tetrahedra $A B C D$ and $A B C E$ is $A B C$ and thus there is a thin line going from $A B C D$ to $A B C E$. Thus there are 10 thin lines corresponding to the number of surrounding triangles. It should be noted that there are two dual link variables $U_{i}$ and $U_{i}^{\prime}$ each of which belongs to the neighboring 4-simplexes separated by the boundary tetrahedron and is located on the same dual link $\tilde{l}_{i}$.

Before getting into the details we briefly figure out how $15-j$ symbols appear after the $d U$ integration. As we have shown in Fig. 3, there are five boundary tetrahedra in a 4-simplex. Each tetrahedron has four triangles and thus there are four thin lines carrying $D$-functions associated to a $d U$ integration. Since each $d U$ integration for the product of four $D$-functions reproduces four $3-j$ symbols, we obtain twenty $3-j$ symbols for each 4 -simplex. Then the ten out of the twenty $3-j$ symbols lead to a 15- $j$ symbols and the rest of the ten 3- $j$ symbols together with those from the neighbouring 4 -simplex reproduce a trivial factor $2 J+1$ attached to each tetrahedron.

We now explicitly show how the $15-j$ symbols appear after the $d U$ integration. 
As a concrete example we focus on the $d U_{1} d U_{1}^{\prime}$ integration on the boundary tetrahedron $B C D E$ of the 4-simplex $A B C D E$. There appear eight $D$-functions in $d U_{1} d U_{1}^{\prime}$ integration,

$$
\begin{aligned}
I_{B C D E}= & \sum_{\left\{k_{i}\right\}} \int d U_{1} d U_{1}^{\prime} D_{m_{1}^{\prime} k_{1}}^{J_{1}}\left(U_{1}^{\prime \dagger}\right) D_{k_{1} m_{1}}^{J_{1}}\left(U_{1}\right) D_{m_{2} k_{2}}^{J_{2}}\left(U_{1}^{\dagger}\right) D_{k_{2} m_{2}^{\prime}}^{J_{2}}\left(U_{1}^{\prime}\right) \\
& \times D_{m_{3}^{\prime} k_{3}}^{J_{3}}\left(U_{1}^{\prime \dagger}\right) D_{k_{3} m_{3}}^{J_{3}}\left(U_{1}\right) D_{m_{4} k_{4}}^{J_{4}}\left(U_{1}^{\dagger}\right) D_{k_{4} m_{4}^{\prime}}^{J_{4}}\left(U_{1}^{\prime}\right)
\end{aligned}
$$

We note that the integration of four $D$-functions leads to the product of four $3-j$ symbols 27],

$$
\begin{aligned}
\int d U \prod_{i=1}^{4} D_{m_{i} n_{i}}^{J_{i}}(U)=\sum_{J, m, n}(2 J & +1)\left(\begin{array}{ccc}
J_{1} & J_{2} & J \\
m_{1} & m_{2} & m
\end{array}\right)(-)^{J+m}\left(\begin{array}{ccc}
J_{3} & J_{4} & J \\
m_{3} & m_{4} & -m
\end{array}\right) \\
& \times\left(\begin{array}{ccc}
J_{1} & J_{2} & J \\
n_{1} & n_{2} & n
\end{array}\right)(-)^{J+n}\left(\begin{array}{ccc}
J_{3} & J_{4} & J \\
n_{3} & n_{4} & -n
\end{array}\right) .
\end{aligned}
$$

Using the above formula and the following relation:

$$
D_{m n}^{J}\left(U^{\dagger}\right)=D_{n m}^{J *}(U)=(-)^{n-m} D_{-n-m}^{J}(U)
$$

we can straightforwardly carry out $d U_{1} d U_{1}^{\prime}$ integration,

$$
\begin{aligned}
I_{B C D E} & =\sum_{I, L, k, l, i}(-)^{k_{1}-m_{1}^{\prime}}(-)^{k_{2}-m_{2}}(-)^{k_{3}-m_{3}^{\prime}}(-)^{k_{4}-m_{4}}(2 L+1)(2 I+1) \\
& \times\left(\begin{array}{ccc}
J_{1} & J_{3} & L \\
m_{1} & m_{3} & l_{1}
\end{array}\right)(-)^{L-l_{1}}\left(\begin{array}{ccc}
L & J_{2} & J_{4} \\
-l_{1} & -m_{2} & -m_{4}
\end{array}\right) \\
& \times\left(\begin{array}{ccc}
J_{1} & J_{3} & L \\
k_{1} & k_{3} & l_{2}
\end{array}\right)(-)^{L-l_{2}}\left(\begin{array}{ccc}
L & J_{2} & J_{4} \\
-l_{2} & -k_{2} & -k_{4}
\end{array}\right) \\
& \times\left(\begin{array}{ccc}
J_{2} & J_{4} & I \\
k_{2} & k_{4} & i_{2}
\end{array}\right)(-)^{I-i_{2}}\left(\begin{array}{ccc}
I & J_{1} & J_{3} \\
-i_{2} & -k_{1} & -k_{3}
\end{array}\right) \\
& \times\left(\begin{array}{ccc}
J_{2} & J_{4} & I \\
m_{2}^{\prime} & m_{4}^{\prime} & i_{1}
\end{array}\right)(-)^{I-i_{1}}\left(\begin{array}{ccc}
I & J_{1} & J_{3} \\
-i_{1} & -m_{1}^{\prime} & -m_{3}^{\prime}
\end{array}\right) .
\end{aligned}
$$

We can evaluate $k_{i}$ summations by using the following formula:

$$
\sum_{m_{1} m_{2}}\left(\begin{array}{ccc}
J_{1} & J_{2} & J \\
m_{1} & m_{2} & m
\end{array}\right)\left(\begin{array}{ccc}
J_{1} & J_{2} & J^{\prime} \\
m_{1} & m_{2} & m^{\prime}
\end{array}\right)=\frac{1}{2 J+1} \delta_{J J^{\prime}} \delta_{m m^{\prime}},
$$

and then obtain

$$
\begin{aligned}
I_{B C D E}=\sum_{L, l, i}(2 L+1) & \left(\begin{array}{ccc}
J_{1} & J_{3} & L \\
m_{1} & m_{3} & l_{1}
\end{array}\right)\left(\begin{array}{ccc}
L & J_{2} & J_{4} \\
-l_{1} & -m_{2} & -m_{4}
\end{array}\right)(-)^{L-l_{1}}(-)^{J_{2}-m_{2}}(-)^{J_{4}-m_{4}} \\
\times & \left(\begin{array}{ccc}
J_{2} & J_{4} & L \\
m_{2}^{\prime} & m_{4}^{\prime} & i_{1}
\end{array}\right)\left(\begin{array}{ccc}
L & J_{1} & J_{3} \\
-i_{1} & -m_{1}^{\prime} & -m_{3}^{\prime}
\end{array}\right)(-)^{L-i_{1}}(-)^{J_{1}-m_{1}^{\prime}}(-)^{J_{3}-m_{3}^{\prime}} .
\end{aligned}
$$


The factor $2 L+1$ can be understood to be associated with the tetrahedron $B C D E$. Two 3- $j$ symbols carrying suffix $m_{i}$ which is assigned to be related to the center of 4-simplex $A B C D E$, will be used to construct the 15- $j$ symbol associated with 4-simplex $A B C D E$, while the other two $3-j$ symbols carrying the suffix $m_{i}^{\prime}$, will be used to construct the $15-j$ symbol associated with the neighboring 4-simplex of $A B C D E$ which share the common tetrahedron $B C D E$.

Hereafter we introduce a graphical method to explain the manipulation of formulae and the derivation leading to the generalized " $15-j$ symbol". The essence of this method is to represent the $3-j$ symbol graphically by trivalent vertex (3-vertex) with a sign factor. The sign is related to the correspondence between the ordering of column in the $3-j$ symbol and right- or left-handed ordering of the angular momentum $J_{i}$ on the trivalent graph. We choose to define the following particular ordering and the sign factor:

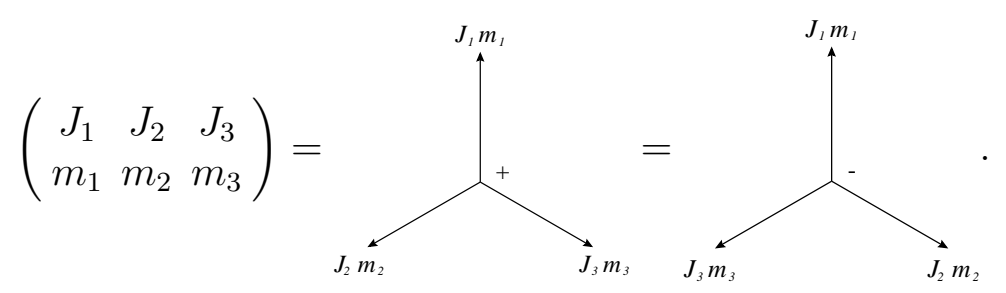

We then define the reversal of the arrows as

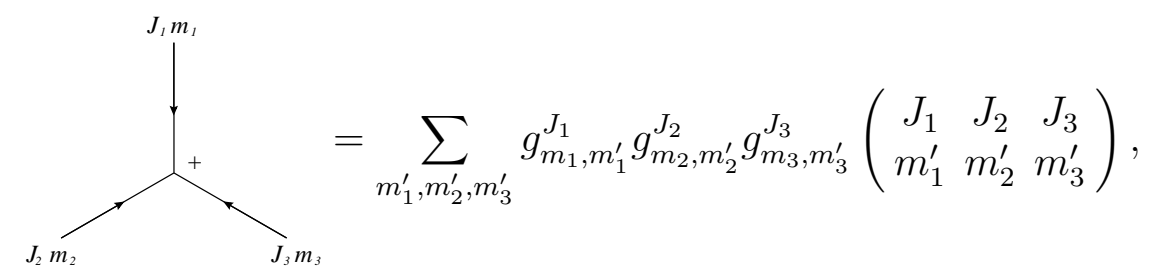

where $g_{m, m^{\prime}}^{J}=\delta_{m,-m^{\prime}}(-1)^{J-m}$ is the $S U(2)$ invariant metric. The following identity

$$
\left(\begin{array}{ccc}
J_{1} & J_{2} & J_{3} \\
m_{1} & m_{2} & m_{3}
\end{array}\right)=\sum_{m_{1}^{\prime}, m_{2}^{\prime}, m_{3}^{\prime}} g_{m_{1}, m_{1}^{\prime}}^{J_{1}} g_{m_{2}, m_{2}^{\prime}}^{J_{2}} g_{m_{3}, m_{3}^{\prime}}^{J_{3}}\left(\begin{array}{ccc}
J_{1} & J_{2} & J_{3} \\
m_{1}^{\prime} & m_{2}^{\prime} & m_{3}^{\prime}
\end{array}\right)
$$

can be graphically represented by

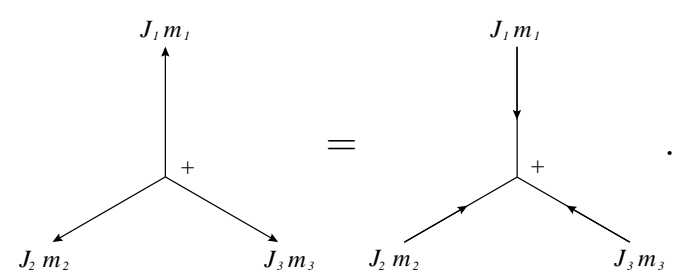

In this way the direction of the arrow is related to the consistent choice of $S U(2)$ invariant metric. For example we can connect two $3-j$ symbols by a metric

$$
\sum_{m, m^{\prime}, m_{3}^{\prime}, m_{4}^{\prime}}\left(\begin{array}{ccc}
J_{1} & J_{2} & J \\
m_{1} & m_{2} & m
\end{array}\right) g_{m, m^{\prime}}^{J} g_{m_{3}, m_{3}^{\prime}}^{J_{3}} g_{m_{4}, m_{4}^{\prime}}^{J_{4}}\left(\begin{array}{ccc}
J & J_{3} & J_{4} \\
m^{\prime} & m_{3}^{\prime} & m_{4}^{\prime}
\end{array}\right)
$$


which is graphically represented by

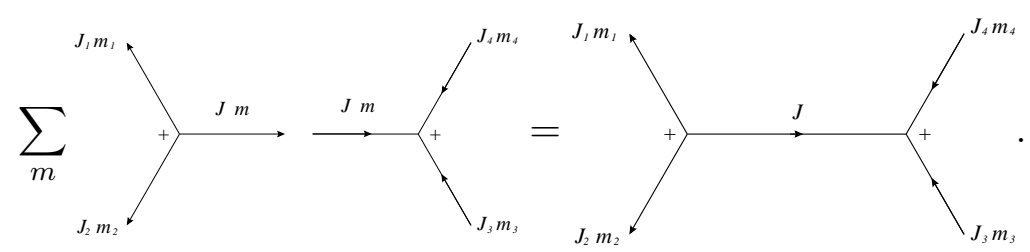

In general any $3 n j$-symbols $(n=2,3,4,5, .$.$) can be graphically represented by$ the closed trivalent graph. For example $6-j$ symbol can be decomposed into four $3-j$ symbols by the formula

$$
\begin{aligned}
& \left\{\begin{array}{lll}
J_{1} & J_{2} & J_{3} \\
J_{4} & J_{5} & J_{6}
\end{array}\right\}=\sum_{\text {all } m_{i}}(-1)^{\sum_{i}\left(J_{i}-m_{i}\right)}\left(\begin{array}{ccc}
J_{1} & J_{2} & J_{3} \\
-m_{1} & -m_{2} & -m_{3}
\end{array}\right) \\
& \times\left(\begin{array}{ccc}
J_{1} & J_{5} & J_{6} \\
m_{1} & -m_{5} & m_{6}
\end{array}\right)\left(\begin{array}{ccc}
J_{4} & J_{2} & J_{6} \\
m_{4} & m_{2} & -m_{6}
\end{array}\right)\left(\begin{array}{ccc}
J_{4} & J_{5} & J_{3} \\
-m_{4} & m_{5} & m_{3}
\end{array}\right),
\end{aligned}
$$

where the factor $(-1)^{\sum_{i}\left(J_{i}-m_{i}\right)}$ can be understood as a sign factor coming from the invariant metric. The graphical presentation of the above $6-j$ symbol can be given
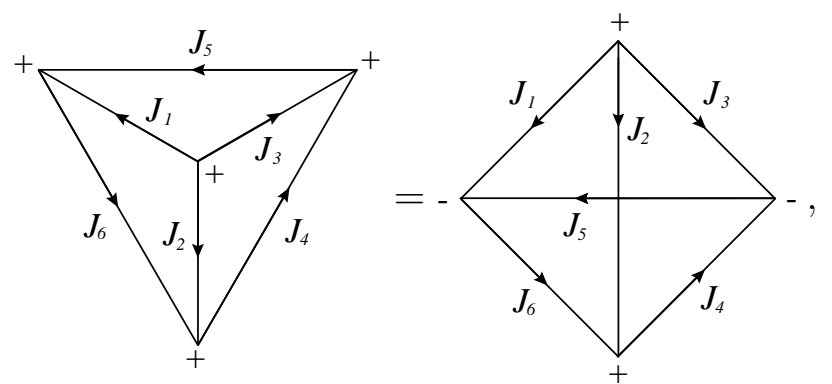

where the rules for the signs on the vertices and the arrows on the lines can be understood. Analogously for more complicated $15-j$ symbols, we need ten $3-j$ symbols and can be represented by closed trivalent graph with ten vertices with sign factors.

Using the graphical method, we can represent the formula (4.12) as

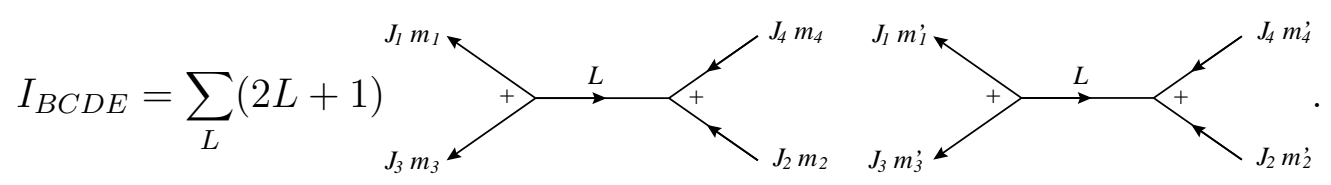

In accordance with (4.12) the factor $(2 L+1)$ and the summation of the magnitude of the angular momentum $L$ are explicitly written while the summation of the third component $l_{1}, i_{1}$ are not written explicitly and should be understood in the graphical representation of the internal line. It should, however, be noted that the above presentation is not the unique expression. There are three other equivalent 
expressions;

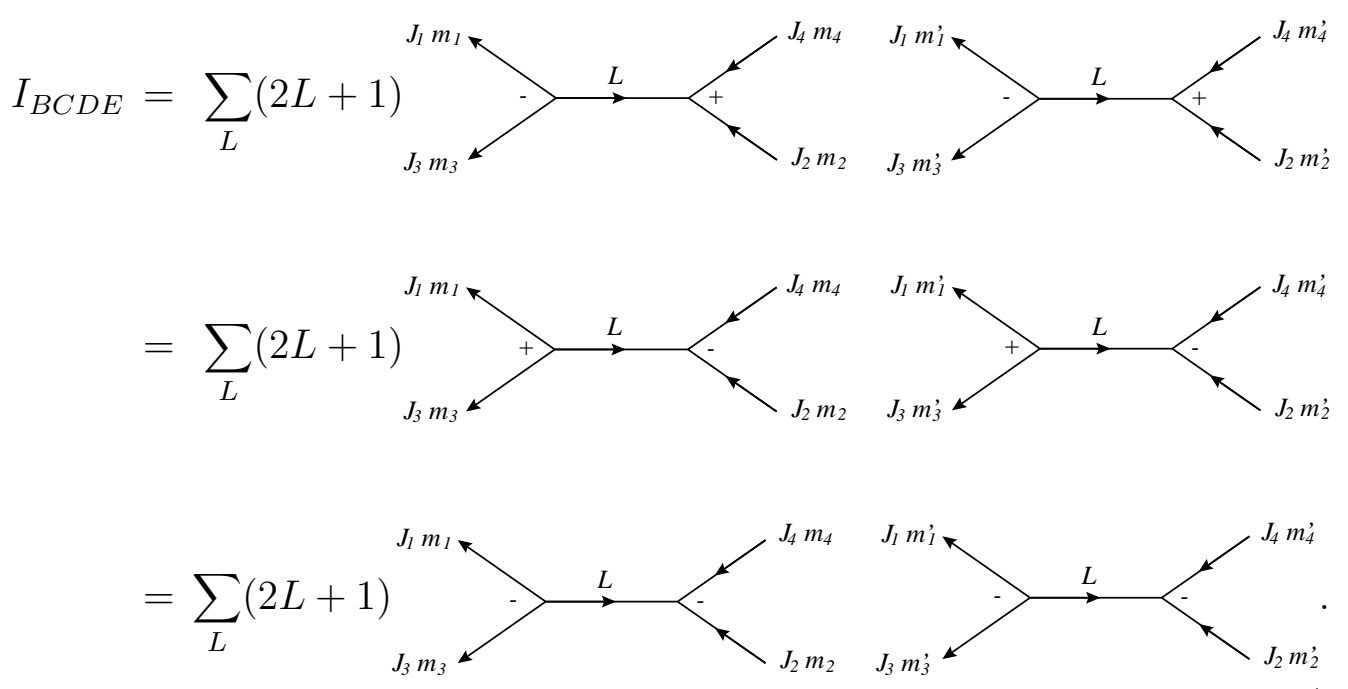

It is important to recognize that the corresponding sign factors are the same in each pair of the four internal line diagrams. In the above particular example we have chosen the dual link integration $d U_{1} d U_{1}^{\prime}$ via the boundary tetrahedron $B C D E$ and obtained the integrated result $I_{B C D E}$. We need to carry out four other dual link integrations; $d U_{2} d U_{2}^{\prime}, d U_{3} d U_{3}^{\prime}, d U_{4} d U_{4}^{\prime}$ and $d U_{5} d U_{5}^{\prime}$ to perform the full dual link integration of the 4-simplex $A B C D E$. They can be carried out in the same way as $d U_{1} d U_{1}^{\prime}$ integration.

Using the formulation explained in the above, we can evaluate the $d U_{i}$ integration graphically,

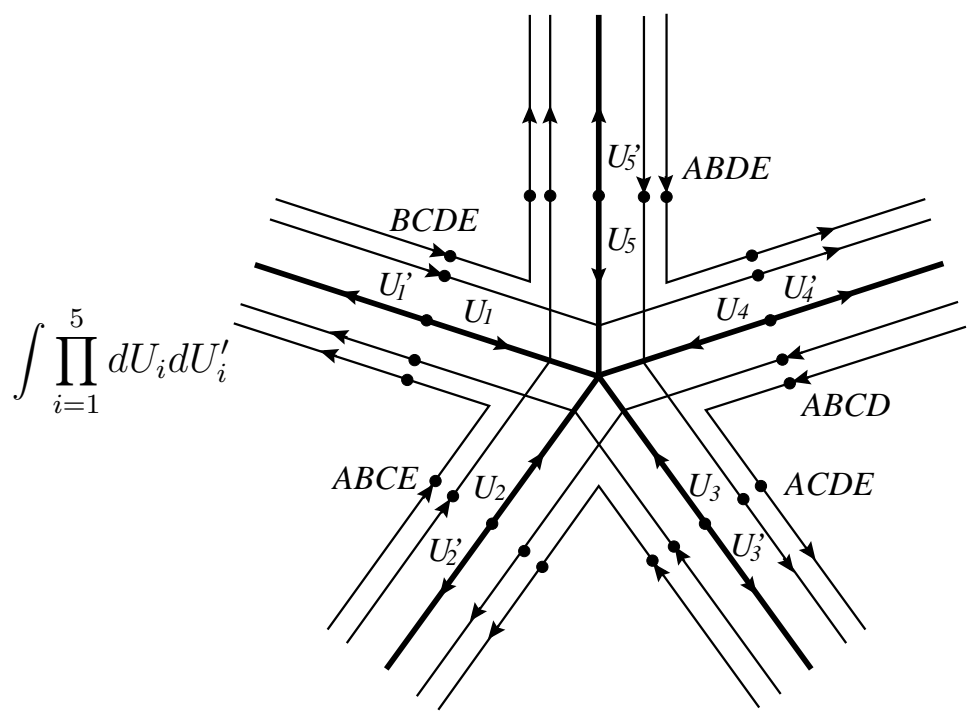




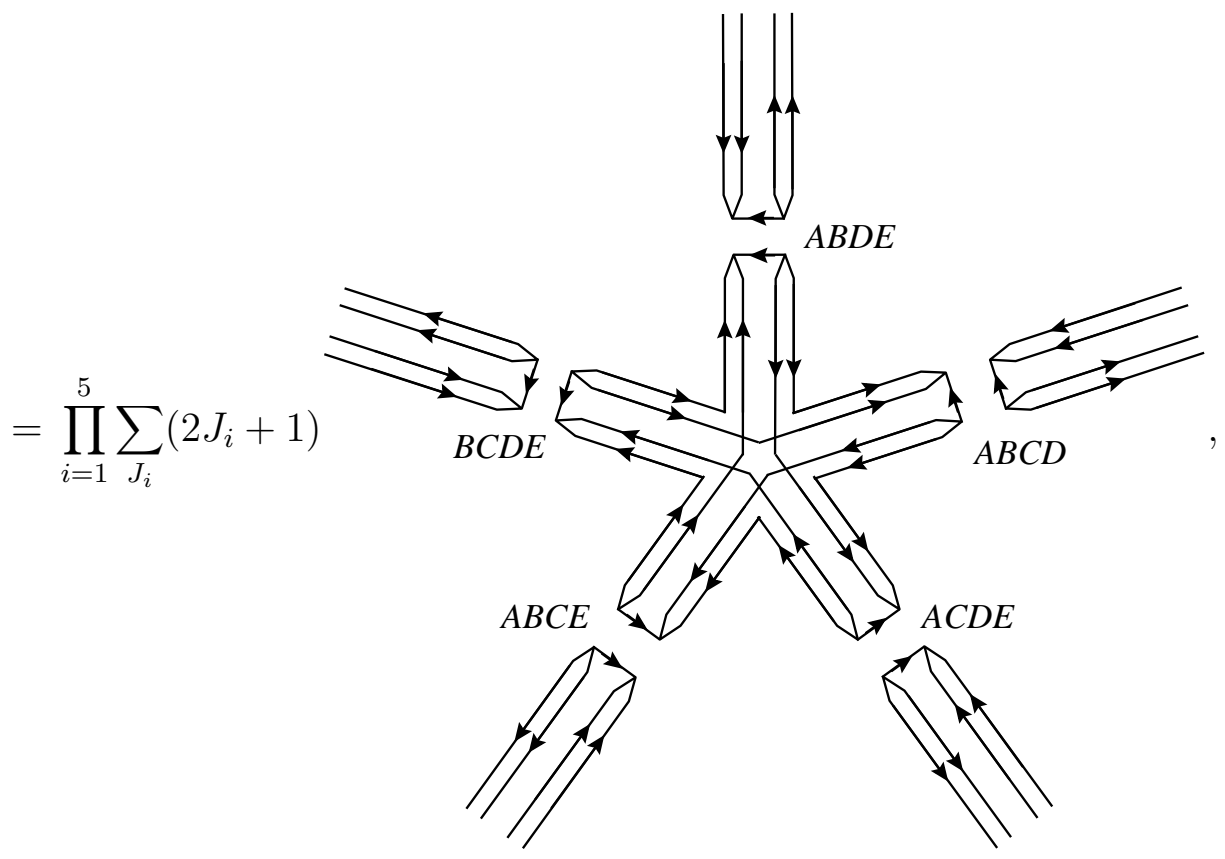

where $J_{1}=J_{B C D E}, J_{2}=J_{A B C E}, J_{3}=J_{A C D E}, J_{4}=J_{A B C D}$ and $J_{5}=J_{A B D E}$. Here $d U_{i} d U_{i}^{\prime}$ integration graphically represented by (4.14) has cut down the thin lines and newly generated a connected line representing the boundary tetrahedron. The closed trivalent graph with ten vertices in the center can be rewritten in topologically equivalent ways,

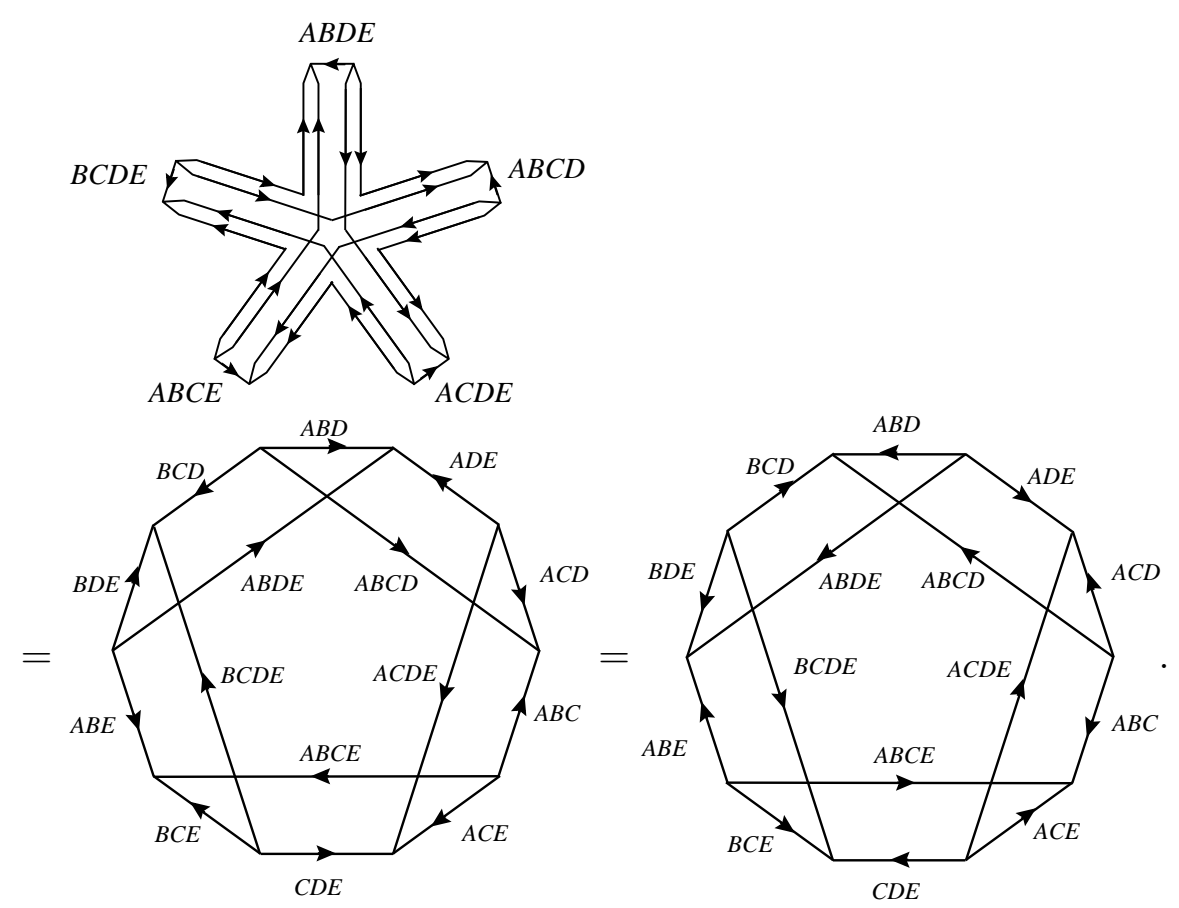

The topological equivalence of the 1st and 2nd graphs are obvious while the 2nd graph equals to the 3rd graph by the following formula:

$$
\sum_{m_{3}}\left(\begin{array}{ccc}
J_{1} & J_{2} & J_{3} \\
m_{1} & m_{2} & m_{3}
\end{array}\right)\left(\begin{array}{ccc}
J_{3} & J_{4} & J_{5} \\
-m_{3} & -m_{4} & -m_{5}
\end{array}\right)(-)^{\sum_{i=3}^{5}\left(J_{i}-m_{i}\right)}
$$




$$
=\sum_{m_{3}}\left(\begin{array}{ccc}
J_{1} & J_{2} & J_{3} \\
-m_{1} & -m_{2} & -m_{3}
\end{array}\right)\left(\begin{array}{ccc}
J_{3} & J_{4} & J_{5} \\
m_{3} & m_{4} & m_{5}
\end{array}\right)(-)^{\sum_{i=1}^{3}\left(J_{i}-m_{i}\right)},
$$

which can be graphically represented by

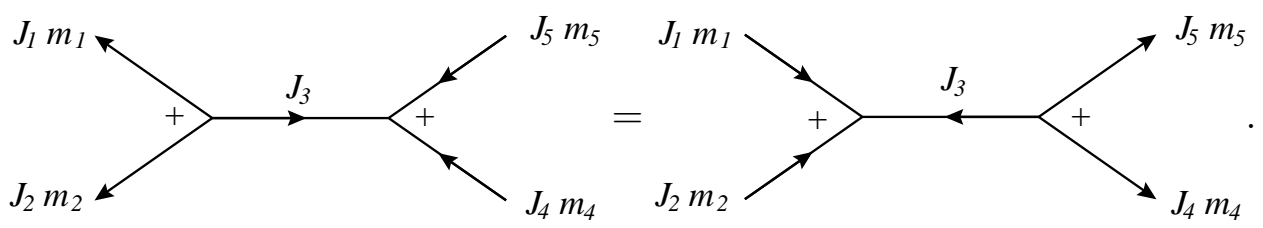

The decuplet graph (4.18) is the definition of our generalized 15- $j$ symbol. There are ten peripheral lines and five internal lines. The internal lines correspond to the tetrahedra which originally come from the one of the internal lines of the corresponding graph in (4.14). The peripheral lines correspond to the common triangle between the neighboring tetrahedra of internal lines. For example the peripheral line $B D E$ is connected with two internal lines $A B D E$ and $B C D E$ and thus corresponds to the common boundary of the two tetrahedra.

We can now obtain the full expression of our partition function in terms of generalized $15-j$ symbols after we carry out $d B$ integration for all the triangles and $d U_{i}$ integration for all the dual links whose dual are the boundary tetrahedra of each 4-simplex,

$$
Z_{L B F}=\sum_{\left\{J_{i}\right\}} \prod_{\text {triangle }}(2 J+1) \prod_{\text {tetrahedron }}(2 J+1) \prod_{4 \text {-simplex }}\{15-j\}
$$

where we have written the $15-j$ symbol symbolically. The factor $(2 J+1)$ attached to a triangle is originated from the same factor as in (4.7) appeared after $d B$ integration on a triangle. The second factor $(2 J+1)$ attached to a tetrahedron is originated from the same factor as in the graph (4.17) appeared after $d U$ integration for each tetrahedron (dual to dual link). Here we have not yet given the explicit form of the generalized $15-j$ symbol with the following reasons. As we have already pointed out in the graphical representation (4.14) and (4.15), there are sign ambiguity for the 3- $j$ representation in the $d U$ integration, which is reflected to the definition of our generalized $15-j$ symbol. In the next section we determine this sign ambiguity by imposing the topological invariance on the partition function $Z_{L B F}$ expressed by the $15-j$ symbols in (4.20). The topological invariance of the partition function on the 4-dimensional simplicial manifold can be assured if the partition function is Pachner move invariant, which we will explain in the next section. 
We conclude this section with a few comments. In the Ooguri's symbolic presentation of the partition function there appear 6 - $j$ symbols in addition to $15-j$ symbols while we don't have this $6-j$ symbols in the partition function. Our definition of the generalized $15-j$ symbol is almost the same as that of Crane-Yetter 16 except for the definition of sign factors.

\section{Pachner Move Invariance of the Partition Func- tion}

In 3-dimensional lattice gravity formulations, it was shown that the lattice ChernSimons gravity leads to the Ponzano-Regge model constructed from $6-j$ symbol 12 which has close correspondence with 3-simplex, tetrahedron. The crucial point of the Ponzano-Regge model is that the particular product of $6-j$ symbols of the model is Alexander move (2-3 move and 1-4 move or equivalently 3-dimensional Pachner move) invariant 9] [10] 11. Since the Ponzano-Regge model is independent how the 3-dimensional simplicial manifold is divided by the moves and thus the model is topological. Then in the 3-dimensional case the continuum limit can be trivially taken and therefore the lattice Chern-Simons gravity action leads to the continuum ISO(3) Chern-Simons gravity action.

In 4 dimensions it is expected that the story proceeds quite parallel as in the 3-dimensional case. Our lattice $B F$ gravity action has led to the partition function constructed from 15-j symbol which has close correspondence with 4-simplex.

As we have already mentioned that any kinds of $15-j$ symbols can be represented by the closed trivalent graph which has ten vertices. Different types of $15-j$ symbols are essentially distinguished by the topology of the graph. For example there are five kinds of standard 15- $j$ symbols which can be decomposed into the product of 6- $j$ and $9-j$ symbols 27. Our generalized $15-j$ symbol graphically given in (4.18) is different from the standard $15-j$ symbol and thus we have called the $15-j$ symbol of (4.18) as "generalized $15-j$ symbol".

In the graphical representation of the generalized $15-j$ symbol, we have not yet specified the sign factors on the trivalent vertices. Here in this section we show that those sign factors are determined by imposing the 4-dimensional Pachner move invariance to the product of the partition function (4.20).

It is the known fact that any 4-dimensional simplicial manifold can be con- 
structed out of 4-dimensional Pachner moves which are composed of $n$ - $m$ moves with $n+m=6$ in 4 dimensions. Thus they include 1-5 move, 2-4 move, 3-3 move and the inverse of the 1-5 and 2-4 moves 28. There are essentially three independent moves; 1-5 move, 2-4 move and 3-3 move to reproduce an arbitrary shape of 4-dimensional simplicial manifold. Those moves are shown in Fig. 4 . In the 1-5 move the center

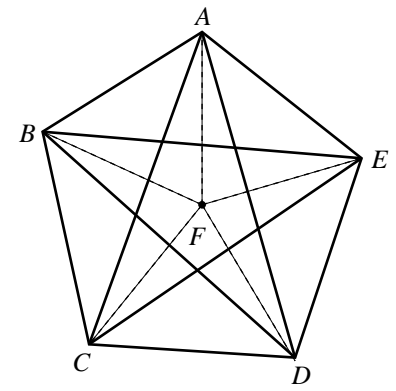

(1)

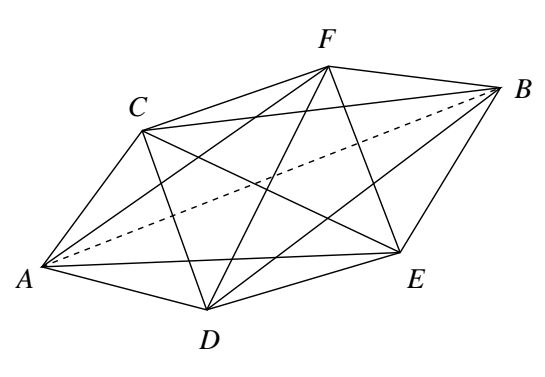

$(2)$

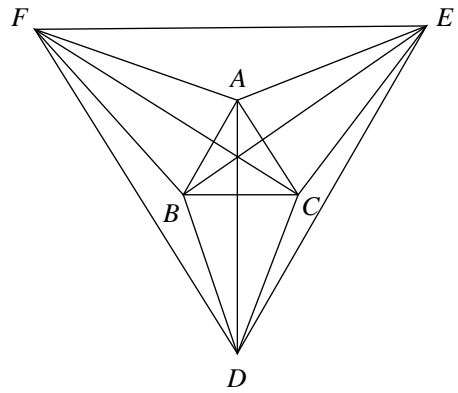

(3)

Figure 4: Pachner moves in 4 dimensions. (1) 1-5 move : $(A B C D E) \rightarrow$ $(B C D E F)(A C D E F)(A B D E F)(A B C E F)(A B C D F), \quad(2) \quad 2-4 \quad$ move : $(A C D E F)(B C D E F) \rightarrow(A B D E F)(A B C E F)(A B C D F)(A B C D E)$, and (3) 3-3 move : $(B C D E F)(A C D E F)(A B D E F) \rightarrow(A B C D E)(A B C E F)(A B C D F)$.

site $F$ in the figure is the common site of final five 4 -simplexes. In the $2-4$ move the dotted link $A B$ is the common link of the final four 4-simplexes. For example in 2-4 move, the initial configuration of a 4-dimensional simplicial manifold including two 4-simplexes $(A C D E F)$ and $(B C D E F)$ will be changed into another simplicial manifold including four 4-simplexes $(A B D E F),(A B C E F),(A B C D F)$ and $(A B C D E)$ after the 2-4 move of Pachner type.

In 3-dimensional case the Alexander move invariance of the Ponzano-Regge model is shown explicitly by several ways [10]11. Since in 4 dimensions the explicit proof of the Pachner move invariance of the partition function of $15-j$ symbol type has not yet been given, we explicitly show the invariance here by a graphical method. There is a proposal of proof of Pachner move invariance by Crane and Yetter who used the similar quantum 15- $j$ symbol as ours but didn't show explicit proof [16]. Furthermore there is some sign factor difference from ours in the definition of $15-j$ symbol. Mathematically there are similar treatments of topologically invariant quantity in 4 dimensions [17].

In order to show the Pachner move invariance of the partition function (4.20) graphically, we need three crucial formulae. The analytic and the corresponding graphical expressions of the formulae are given in the following. We may call an 
expression to be closed if all the third components of the angular momentum are summed up. Then if there are two closed expressions each of which has two $3-j$ symbols with the common magnitude of angular momentum, they can be connected into one closed expression as follows:

$$
\begin{gathered}
\sum_{J}(2 J+1) \sum_{m_{i} M m_{i}^{\prime} M^{\prime}}\left(\begin{array}{ccc}
j_{1} & j_{2} & J \\
m_{1} & m_{2} & M
\end{array}\right)\left\langle j_{1} m_{1} j_{2} m_{2}|\mathcal{M}| j_{3} m_{3}^{\prime} j_{4} m_{4}^{\prime}\right\rangle\left(\begin{array}{ccc}
j_{3} & j_{4} & J \\
-m_{3}^{\prime} & -m_{4}^{\prime} & -M
\end{array}\right) \\
\times\left(\begin{array}{ccc}
j_{3} & j_{4} & J \\
m_{3} & m_{4} & M^{\prime}
\end{array}\right)\left\langle j_{3} m_{3} j_{4} m_{4}|\mathcal{N}| j_{1} m_{1}^{\prime} j_{2} m_{2}^{\prime}\right\rangle\left(\begin{array}{ccc}
j_{1} & j_{2} & J \\
-m_{1}^{\prime} & -m_{2}^{\prime} & -M^{\prime}
\end{array}\right) \\
\times(-)^{\sum_{i=1}^{4}\left(j_{i}-m_{i}^{\prime}\right)+(J-M)+\left(J-M^{\prime}\right)} \\
=\sum_{m_{i}}\left\langle j_{1} m_{1} j_{2} m_{2}|\mathcal{M}| j_{3} m_{3} j_{4} m_{4}\right\rangle\left\langle j_{3} m_{3} j_{4} m_{4}|\mathcal{N}| j_{1} m_{1} j_{2} m_{2}\right\rangle,
\end{gathered}
$$

which is graphically given by

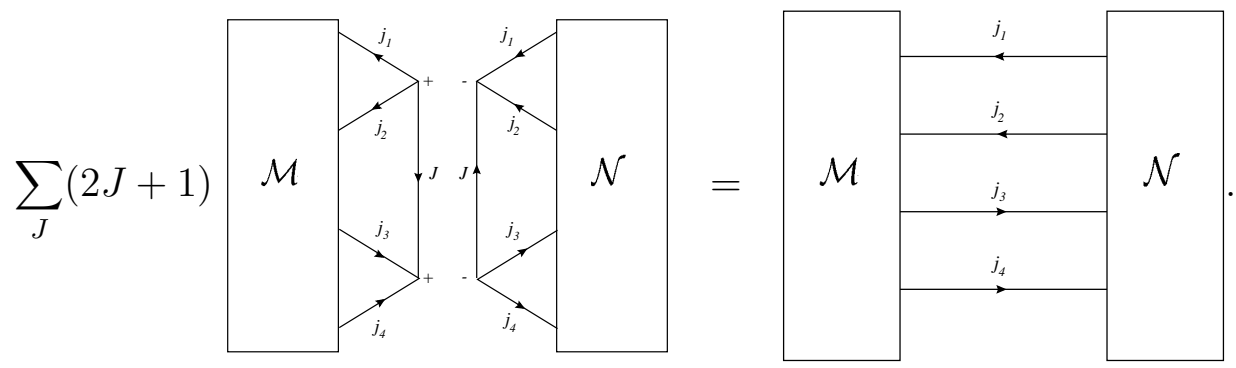

The orthogonality of $3-j$ symbol with a weight factor is

$$
\sum_{J m^{\prime}}(2 J+1)\left(\begin{array}{ccc}
J_{1} & J_{2} & J \\
m_{1} & m_{2} & m^{\prime}
\end{array}\right)\left(\begin{array}{ccc}
J_{1} & J_{2} & J \\
-m_{1}^{\prime} & -m_{2}^{\prime} & -m^{\prime}
\end{array}\right)(-)^{\sum_{i=1}^{3}\left(J_{i}-m_{i}^{\prime}\right)}=\delta_{m_{1} m_{1}^{\prime}} \delta_{m_{2} m_{2}^{\prime}},
$$

which is graphically given by

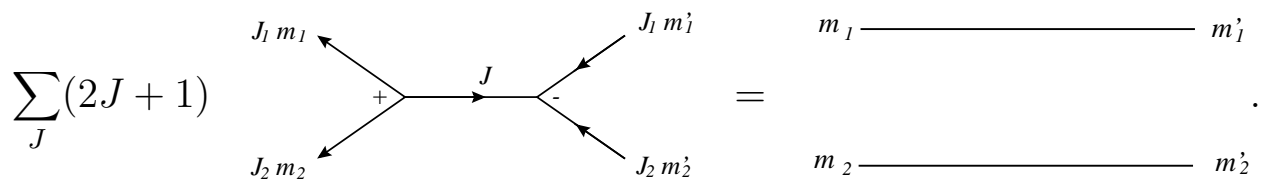

The orthogonality of $3-j$ symbol with two angular momentum magnitudes summing up leads

$$
\begin{aligned}
& \sum_{J_{2} m_{2}^{\prime} J_{3} m_{3}^{\prime}}\left(2 J_{2}+1\right)\left(2 J_{3}+1\right)\left(\begin{array}{ccc}
J_{1} & J_{2} & J_{3} \\
m_{1} & m_{2}^{\prime} & m_{3}^{\prime}
\end{array}\right)\left(\begin{array}{ccc}
J_{1} & J_{2} & J_{3} \\
m_{1}^{\prime} & m_{2}^{\prime} & m_{3}^{\prime}
\end{array}\right)(-)^{\sum_{i=1}^{3}\left(J_{i}-m_{i}^{\prime}\right)} \\
= & \Lambda \cdot \delta_{m_{1} m_{1}^{\prime}}
\end{aligned}
$$

where $\Lambda$ is the infinite constant which needs a regularization and is the same factor appeared in the Ponzano-Regge model. Here we introduce the following cut-off 
factor of the angular momentum to regularize the infinite constant

$$
\begin{aligned}
\Lambda(\lambda)= & \frac{1}{2 J_{1}+1} \sum_{\substack{K_{2}, K_{3} \leq \lambda,\left|K_{2}-K_{3}\right| \leq J_{1} \leq K_{2}+K_{3}}}\left(2 K_{2}+1\right)\left(2 K_{3}+1\right) \\
= & \sum_{J=0}^{\lambda}(2 J+1)^{2} \sim \frac{4 \lambda^{3}}{3}(\lambda \rightarrow \infty) .
\end{aligned}
$$

Then (5.3) can be graphically given by

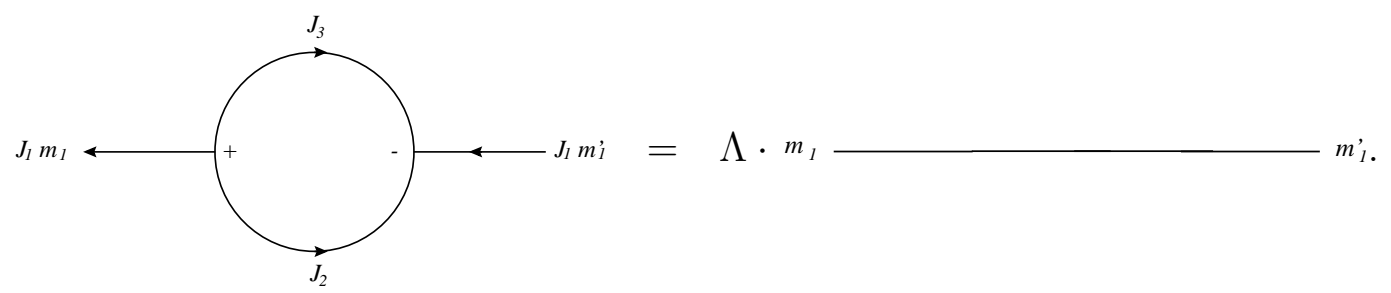

An example of the combined graphical relation of (5.1), (5.2) and (5.3) is

$$
\begin{aligned}
I= & \sum_{J_{1} J_{2} J_{3}} \prod_{i=1}^{3}\left(2 J_{i}+1\right) \sum_{m_{1} m_{2} n_{1} n_{3}} \\
& \times\left(\begin{array}{ccc}
J_{1} & J_{3} & J_{4} \\
m_{1} & n_{3} & m_{4}
\end{array}\right)\left(\begin{array}{ccc}
J_{1} & J_{5} & J_{2} \\
-m_{1} & -m_{5} & -m_{2}
\end{array}\right)(-)^{\sum_{i=1,2,5}\left(J_{i}-m_{i}\right)} \\
& \times\left(\begin{array}{ccc}
J_{1} & J_{5} & J_{2} \\
n_{1} & n_{5} & m_{2}
\end{array}\right)\left(\begin{array}{ccc}
J_{1} & J_{3} & J_{4} \\
-n_{1} & -n_{3} & -n_{4}
\end{array}\right)(-)^{\sum_{i=1,3,4}\left(J_{i}-n_{i}\right)},
\end{aligned}
$$

which is graphically given by

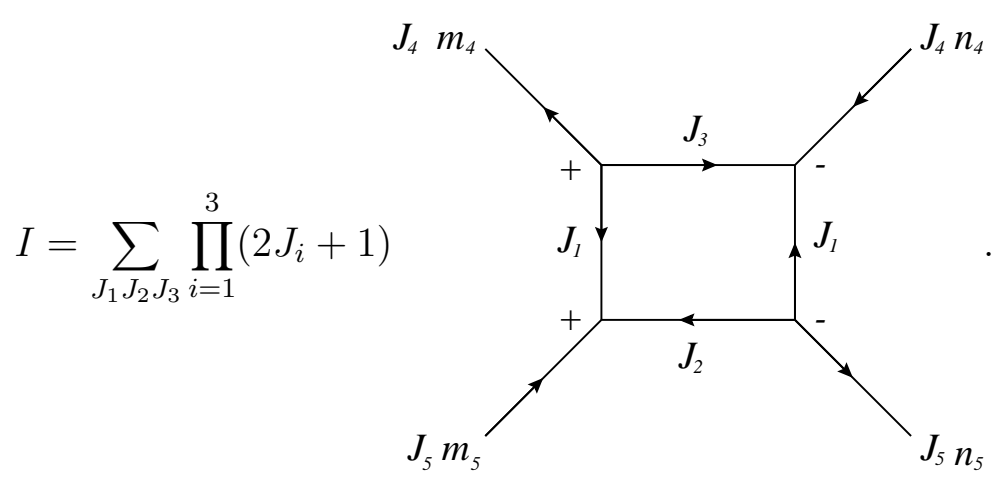

Using the relations (5.2) and (5.3), we obtain

$$
\begin{aligned}
I= & \sum_{J_{1} J_{3}}\left(2 J_{1}+1\right)\left(2 J_{3}+1\right) \\
& \times \sum_{n_{1} n_{3}}\left(\begin{array}{ccc}
J_{1} & J_{3} & J_{4} \\
n_{1} & n_{3} & m_{4}
\end{array}\right)\left(\begin{array}{ccc}
J_{1} & J_{3} & J_{4} \\
-n_{1} & -n_{3} & -n_{4}
\end{array}\right)(-)^{\sum_{i=1,3,4}\left(J_{i}-n_{i}\right)} \cdot \delta_{m_{5} n_{5}} \\
= & \Lambda \delta_{m_{4}, n_{4}} \delta_{m_{5} n_{5}},
\end{aligned}
$$


which is graphically given by

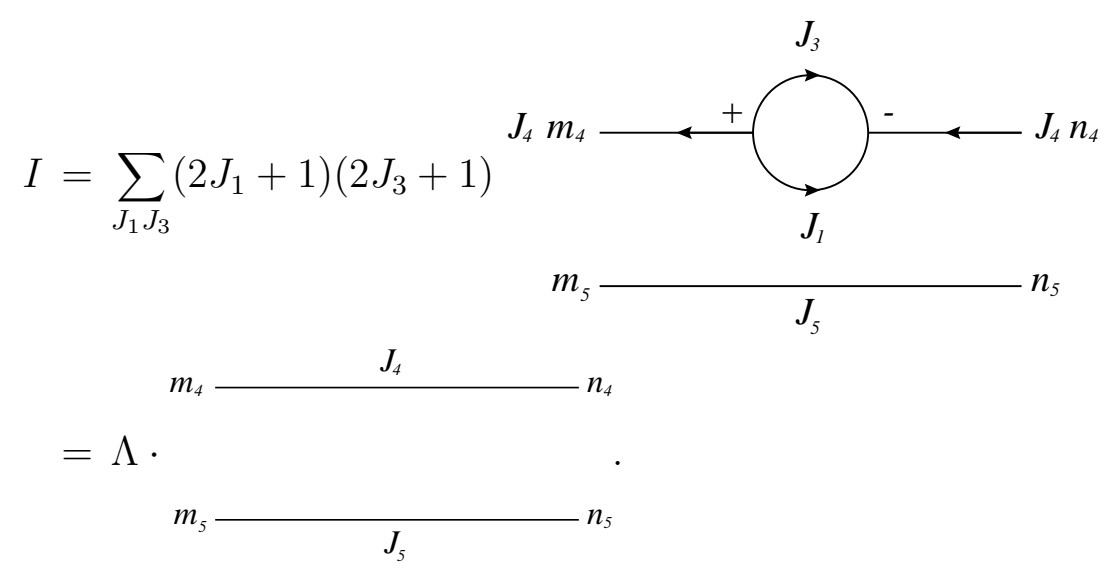

We now explicitly show the invariance of the partition function (4.20) under the 4-2 Pachner move graphically by using the above formulae. The 4-2 move is simply the inverse of 2-4 move and includes all the necessary manipulations. The 5-1 and 3-3 Pachner move invariance can be proved in the similar way. We will abbreviate the summation of $J$ and factors $(2 J+1)$ in the following graphical calculation.

We first display four decuplet diagrams corresponding to the four 4-simplexes in particular rule. We first note that the boundary of the initial four 4-simplexes should coincide with the boundary of the final two simplexes with correct orientations. With the help of homological algebraic notations, we obtain the following relation:

$$
\begin{aligned}
& \partial(A B C D E)-\partial(A B C D F)-\partial(A B D E F)+\partial(A B C E F) \\
= & \partial(B C D E F)-\partial(A C D E F) \\
= & B C D E-A C D E-B D E F+A D E F \\
& -B C D F+A C D F+B C E F-A C E F,
\end{aligned}
$$

where the boundary operator $\partial$ is defined by

$$
\partial(A B C D E)=B C D E-A C D E+A B D E-A B C E+A B C D .
$$

In the first row of the decuplet diagrams in the following first equation, we have shown the 4-simplex $A B C D E$ where the internal lines corresponding to tetrahedra are arranged in the order starting from $B C D E$ and then $A C D E$ with common triangle $C D E$ and so on as in the order of (5.10). In the second row of the first decuplet diagrams, we show the 4-simplex $A B C D F$ with the opposite arrows with respect to the diagram $A B C D E$ reflecting the sign difference in (5.9). For example the arrow of $B C D E$ for the 4-simplex $A B C D E$ in (1) is the opposite to the arrow 
of $B C D F$ for the 4-simplex $A B C D F$ in (2), which are located on the same position of each decouplet. We then display the 4-simplexes $A B D E F$ and $A B C E F$ with the same rule in the first column of the equations. 
(1)

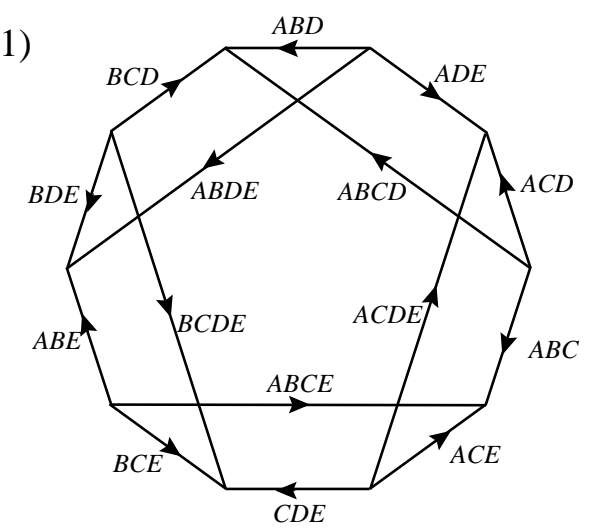

(2)

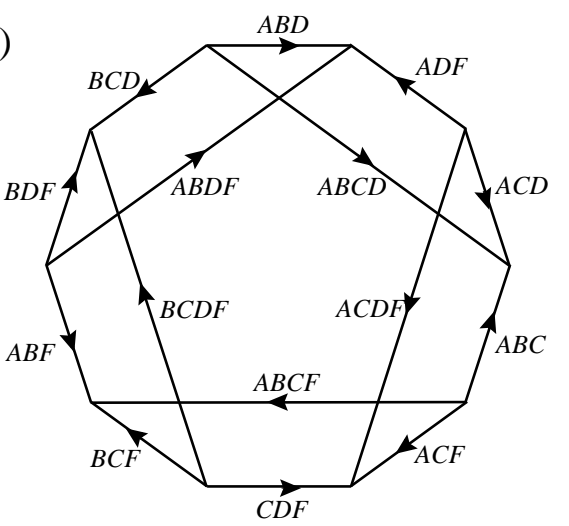

(3)

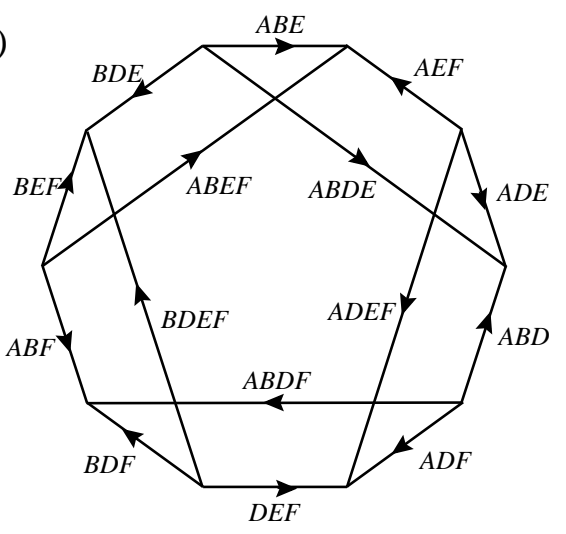

(4)

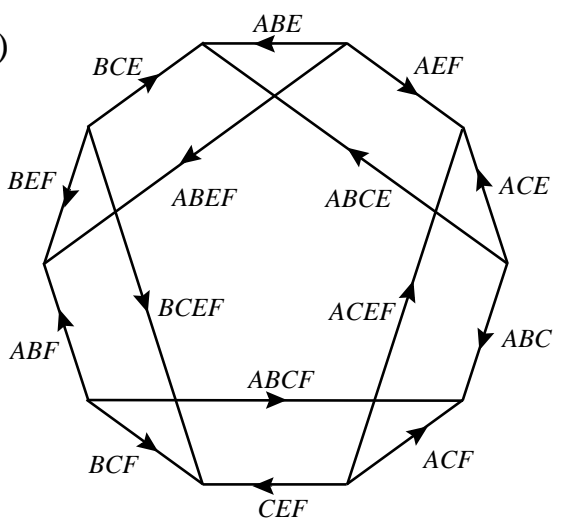

(5)

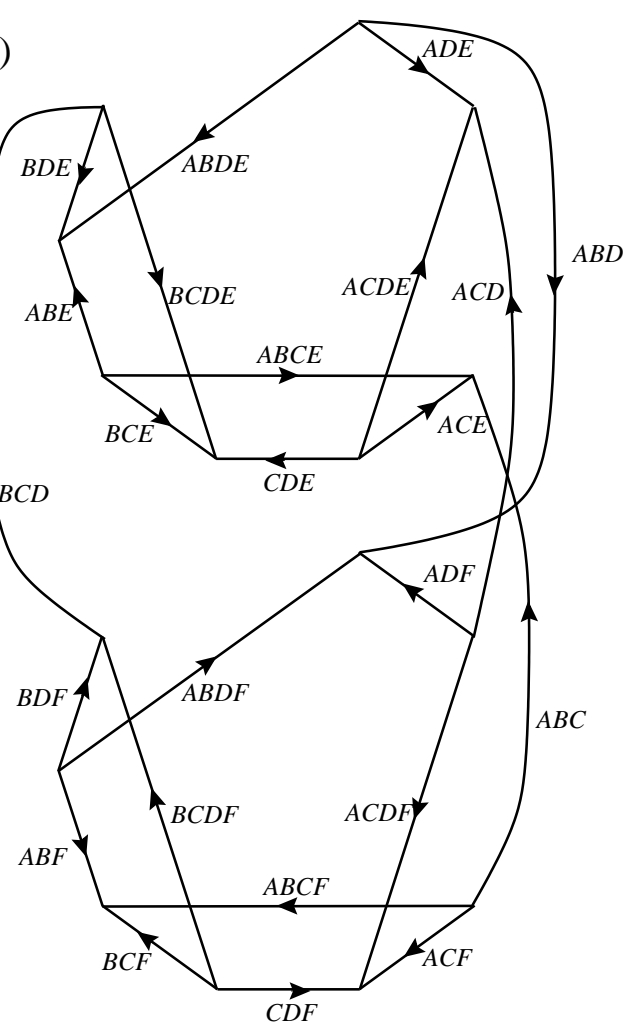

(6)

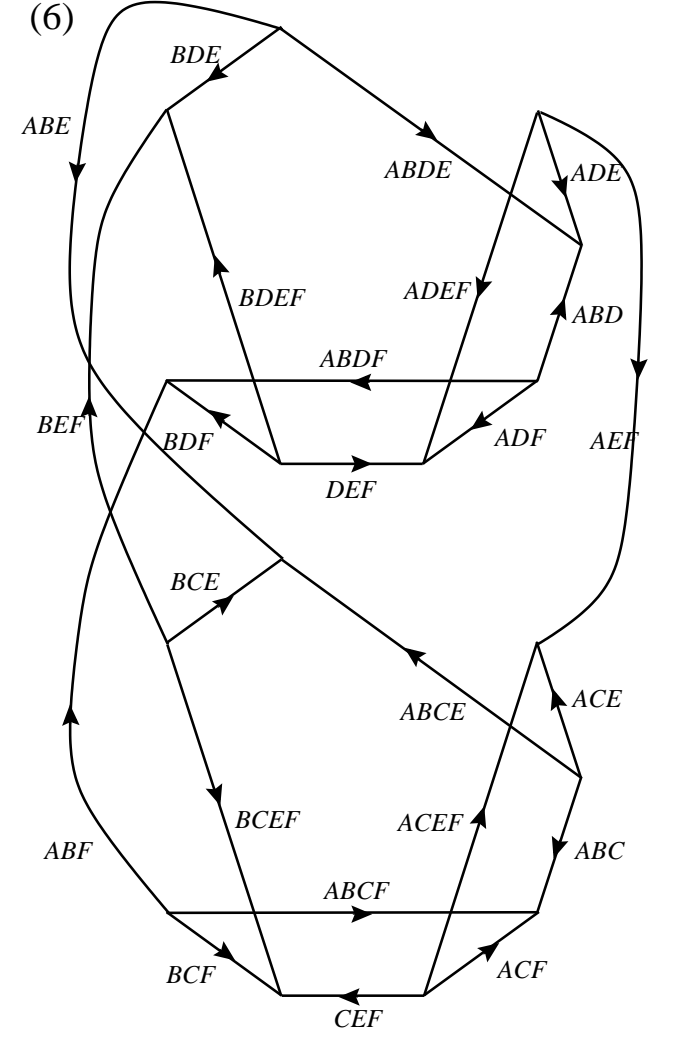



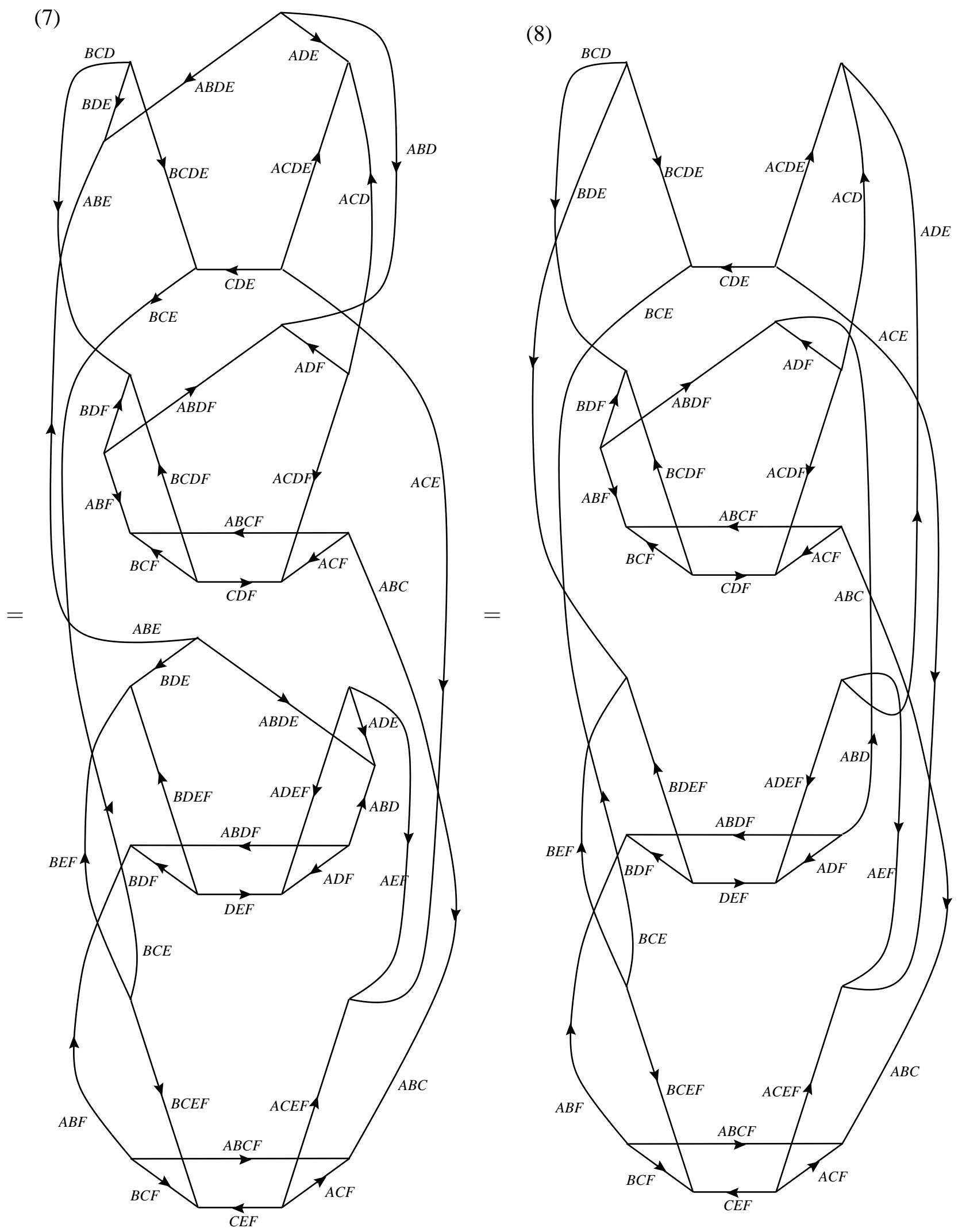

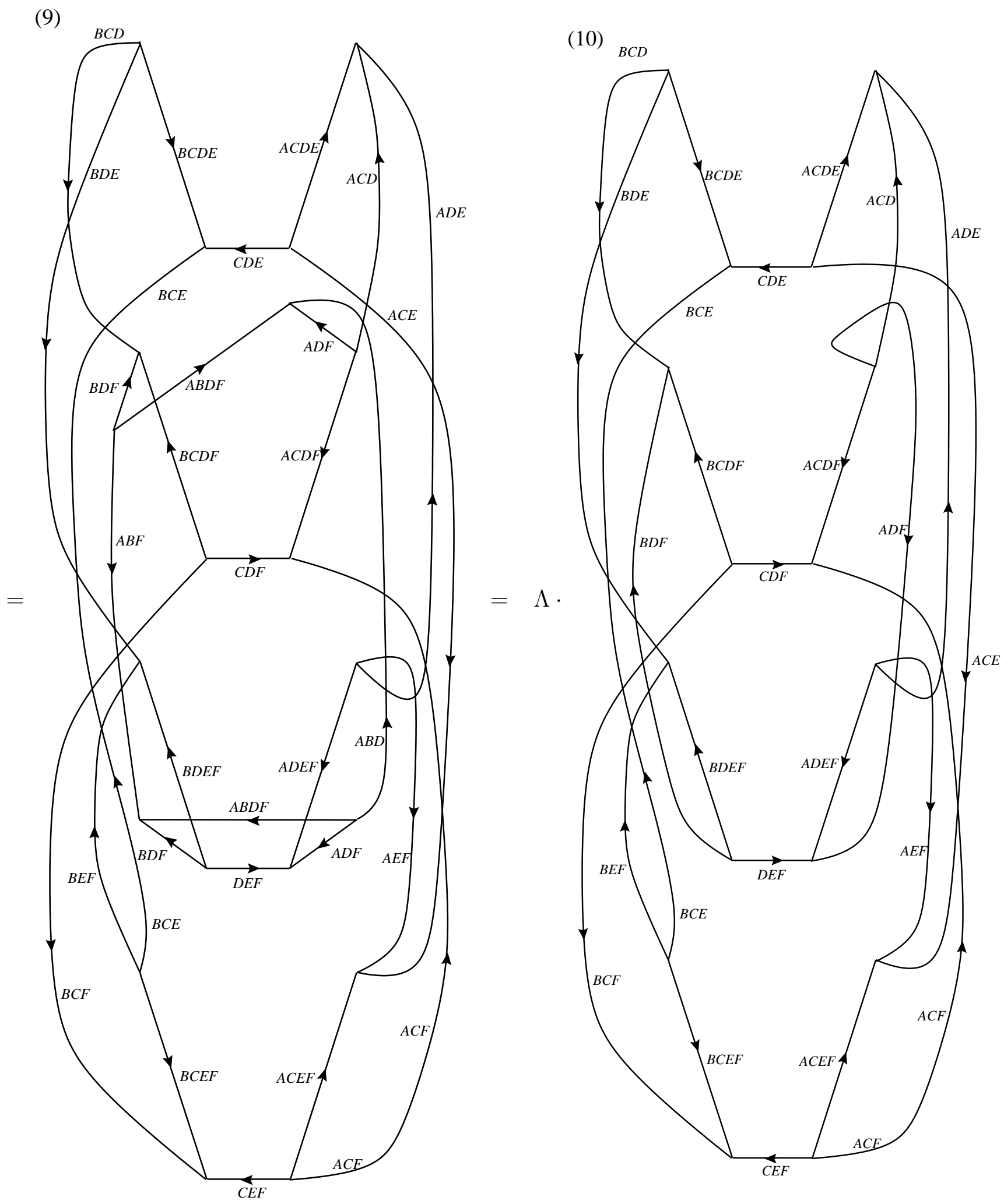

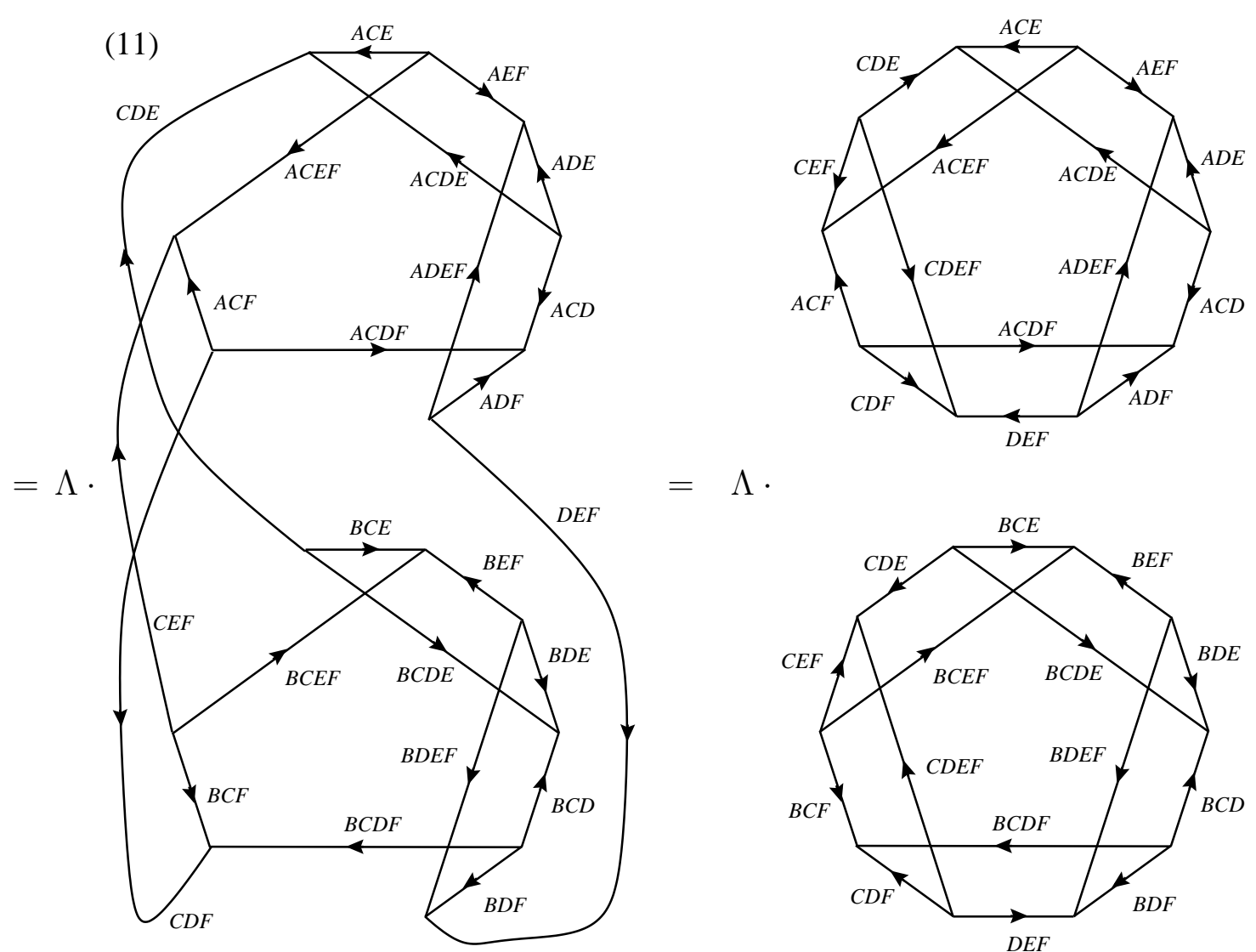

In the first equality we have used the formula (5.1) for the internal lines of tetrahedra $A B C D$ and $A B E F$ to combine two diagrams (1) and (2) into (5) and (3) and (4) into (6), respectively. In the second equality we have again used the formula (5.1) for the common internal lines $A B C E$ to combine the diagrams (5) and (6) into (7). In the third equality, we have used the formula (5.2) to get rid of $A B E$ and then $A B D E$ successively in (7) and reached the diagram (8). Similarly in the forth equality $A B C$ and $A B C F$ are removed from (8) into (9). In the fifth equality we have used the relations (5.6) and (5.8) since there is a closed loop with two internal tetrahedra; $A B D F \rightarrow A B D \rightarrow A B D F \rightarrow A B F \rightarrow A B D F$ in the diagram (9). In the sixth equality we rearranged the diagram (10) in the form of diagram (11). In the last equality we have used again the formula (5.1) in the inverse order to reproduce the internal line of tetrahedron $C D E F$.

We have thus shown that the product of initial four decuplet diagrams is equivalent to the product of the final two decuplet diagrams multiplied by the regularized constant $\Lambda$. Thus the product of the $15-j$ symbols of the partition function (4.20) can be decomposed into another product by the 4-2 move. The proof of 5-1 and 3-3 move invariance of the partition function proceeds quite parallel to the proof of 4-2 
move. In the treatment of 5-1 move, we actually obtain the regularization factor $\Lambda^{4}$, while 3-3 move does not reproduce any regularization factor.

We first note that in 4-2 move there is a common link for the initial four 4simplexes while there is a common site for the initial five 4-simplexes in 5-1 move. We then ask how many powers of $\Lambda$ we need to be compatible with the Pachner moves. We first count the change of the number of $i$-simplexes $\Delta N_{i}$ before and after the Pachner moves. We list the result in Table 1 .

\begin{tabular}{|c|c|c|c|c|c|}
\hline & $\Delta N_{0}$ & $\Delta N_{1}$ & $\Delta N_{2}$ & $\Delta N_{3}$ & $\Delta N_{4}$ \\
\hline 5-1 move & 1 & 5 & 10 & 10 & 4 \\
\hline 4-2 move & 0 & 1 & 4 & 5 & 2 \\
\hline 3-3 move & 0 & 0 & 0 & 0 & 0 \\
\hline
\end{tabular}

Table 1: The difference of the number of simplexes before and after the Pachner moves.

Since we have obtained $\Lambda^{4}$ in 5-1 move and $\Lambda$ in 4-2 move, we need to obtain the following $a_{i}$ satisfying:

$$
\begin{aligned}
a_{0}+5 a_{1}+10 a_{2}+10 a_{3}+4 a_{4} & =4, \\
a_{1}+4 a_{2}+5 a_{3}+2 a_{4} & =1 .
\end{aligned}
$$

Then the total power of $\Lambda$ of the 4-dimensional simplicial manifold in consideration will be given by

$$
\Lambda \sum_{i=0}^{4} a_{i} N_{i}
$$

There are the following well known relations among the total number of $i$-simplex $N_{i}$ in 4-dimensional simplicial manifold[29]:

$$
\begin{aligned}
N_{0}-N_{1}+N_{2}-N_{3}+N_{4} & =\chi \\
2 N_{1}-3 N_{2}+4 N_{3}-5 N_{4} & =0 \\
2 N_{3} & =5 N_{4},
\end{aligned}
$$

where $\chi$ is Euler number. Solving $N_{2}, N_{3}$ and $N_{4}$ in terms of $N_{0}$ and $N_{1}$ in (5.13), we obtain the power of $\Lambda$ as

$$
\sum_{i=0}^{4} a_{i} N_{i}=N_{0}\left(a_{0}-10 a_{2}-15 a_{3}-6 a_{4}\right)+N_{1}\left(a_{1}+4 a_{2}+5 a_{3}+2 a_{4}\right)+\chi\left(10 a_{2}+15 a_{3}+6 a_{4}\right) .
$$


As far as $a_{i}$ satisfies the relation (5.11), we can arbitrarily take the integer $a_{i}$. Taking the choice $a_{2}=a_{3}=a_{4}=0$, we obtain Euler number independent solution of the $\Lambda$ power dependence,

$$
\Lambda^{-N_{0}+N_{1}}
$$

To summarize we obtain the Pachner move invariant partition function,

$$
Z_{L B F}=\sum_{\left\{J_{i}\right\}} \prod_{\text {site }} \Lambda^{-1} \prod_{\text {link }} \Lambda \prod_{\text {triangle }}(2 J+1) \prod_{\text {tetrahedron }}(2 J+1) \prod_{4 \text {-simplex }}\{15-j\} .
$$

Finally we give arguments to determine the sign factors of the $15-j$ symbols. We first note that the decuplet graph of our 15- $j$ symbol with any given sign factor configuration is equal to the one that has reversed all sign factors,

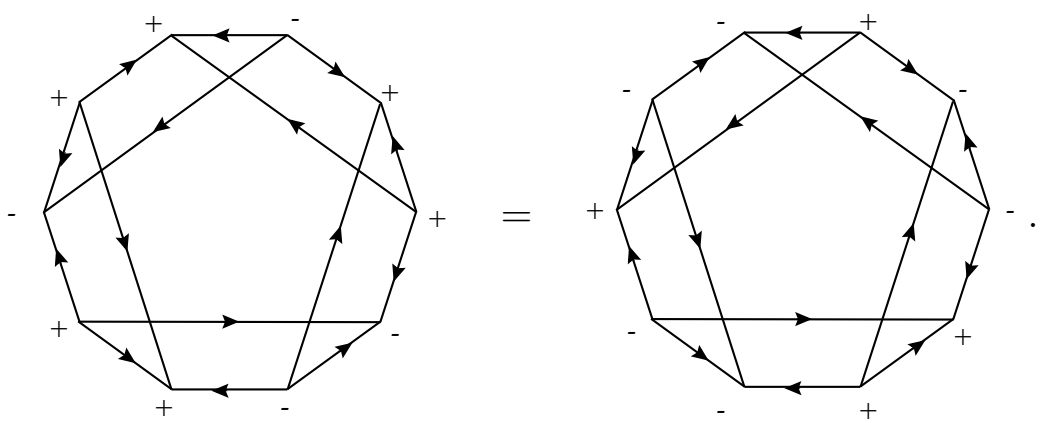

We can thus take the sign factor of one particular vertex + without loss of generality. We then take the following special choice of one sign factor for the initial four 4simplexes of 4-2 move:
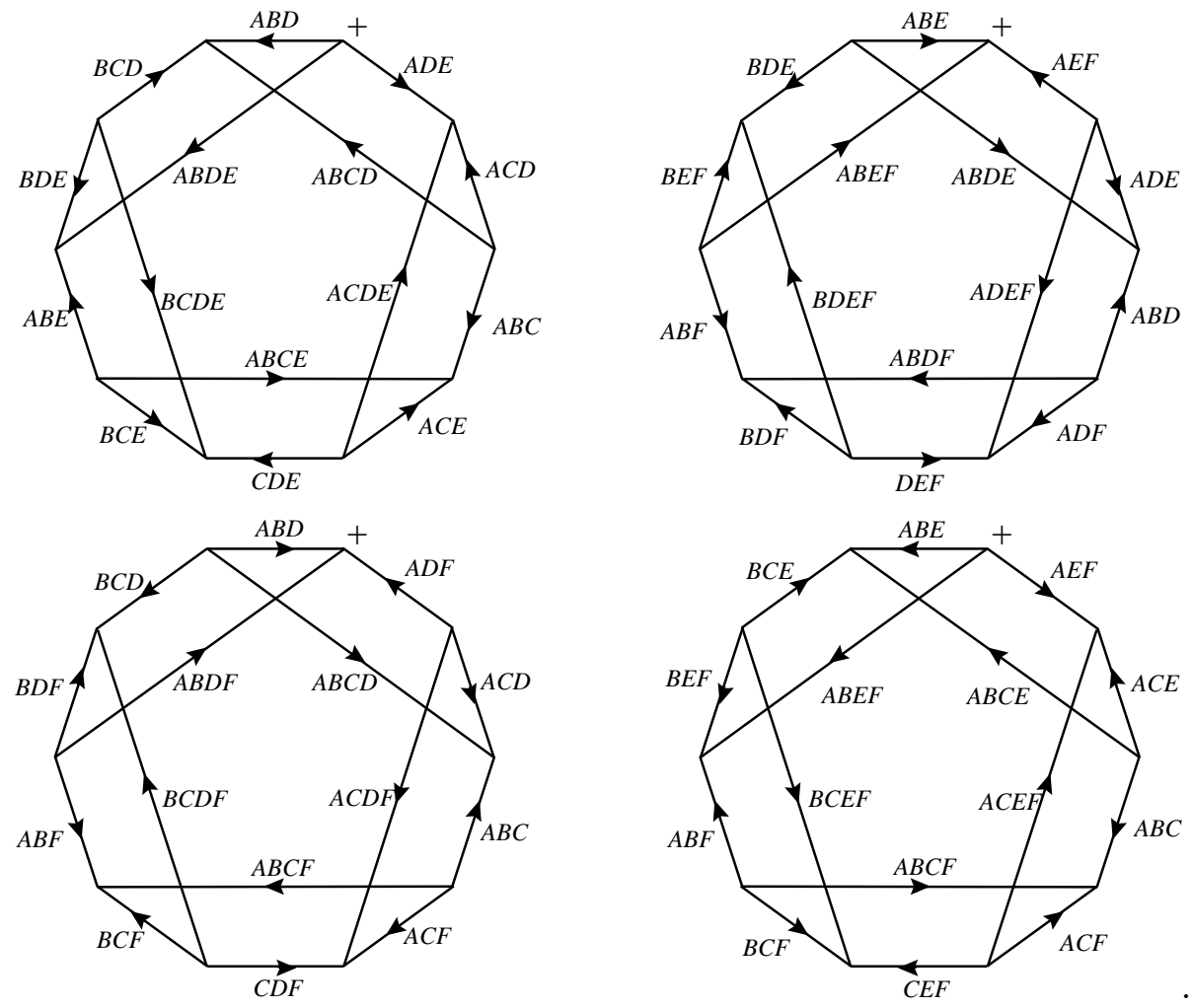
When we consider an arbitrary 4-dimensional simplicial manifold composed of 4-simplexes, any of two neighboring 4-simplexes have one boundary tetrahedron in common. In the corresponding expression of this simplicial manifold by the generalized 15- $j$ symbols, there are thus always the same pair of internal lines in the decuplet diagrams. As we have already mentioned in (4.14) and (4.15), the sign factors of two vertices associated to the same tetrahedron should be the same. Using this fact, we can determine the other sign factors of initial four 4-simplexes of 4-2 move up to the five unknown sign ambiguities denoted by $\alpha, \beta, \gamma, \delta$ and $\epsilon$
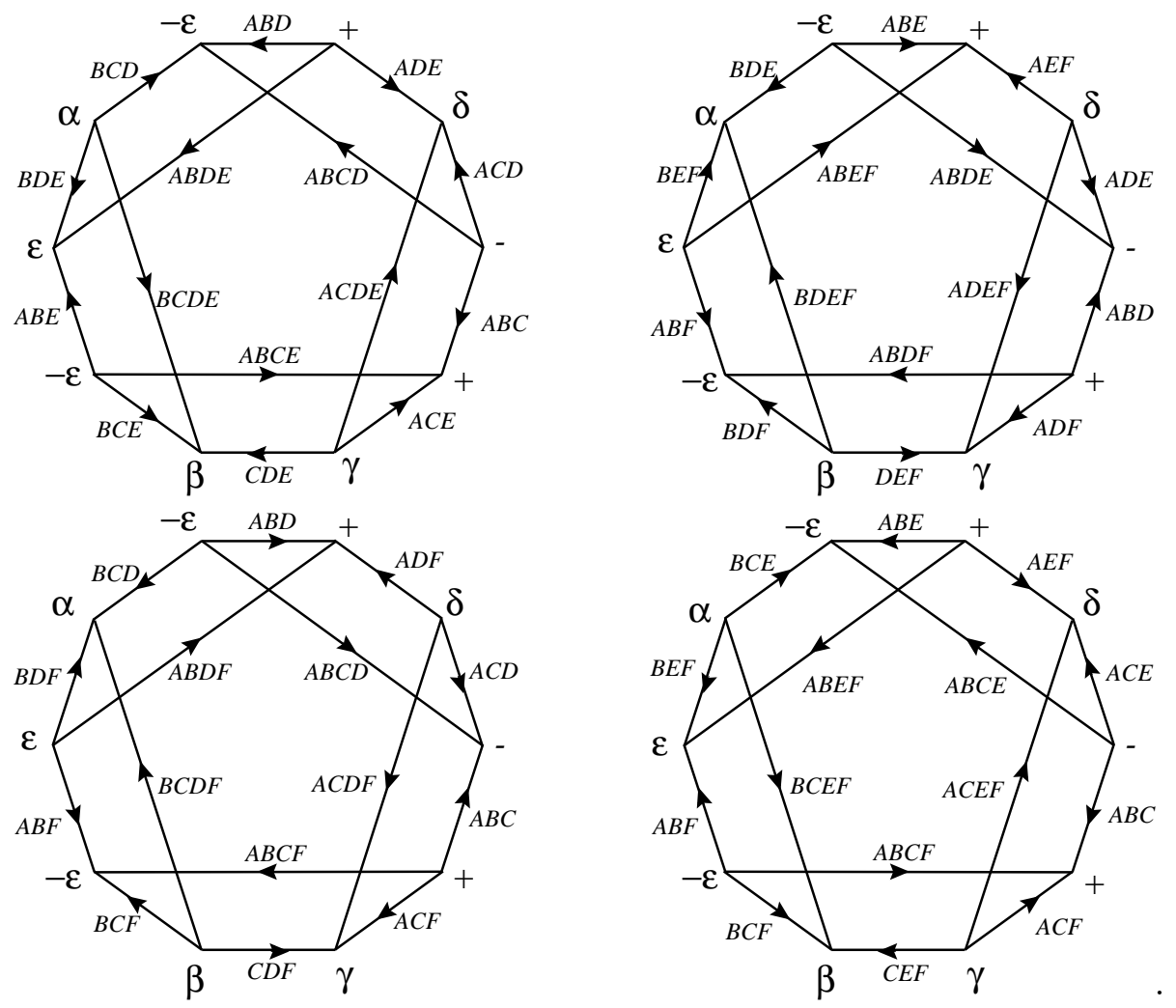

The final possible sign configuration of two 4-simplexes in 4-2 move can be found by tracing the graphical manipulations and identifying the signs of common vertices,
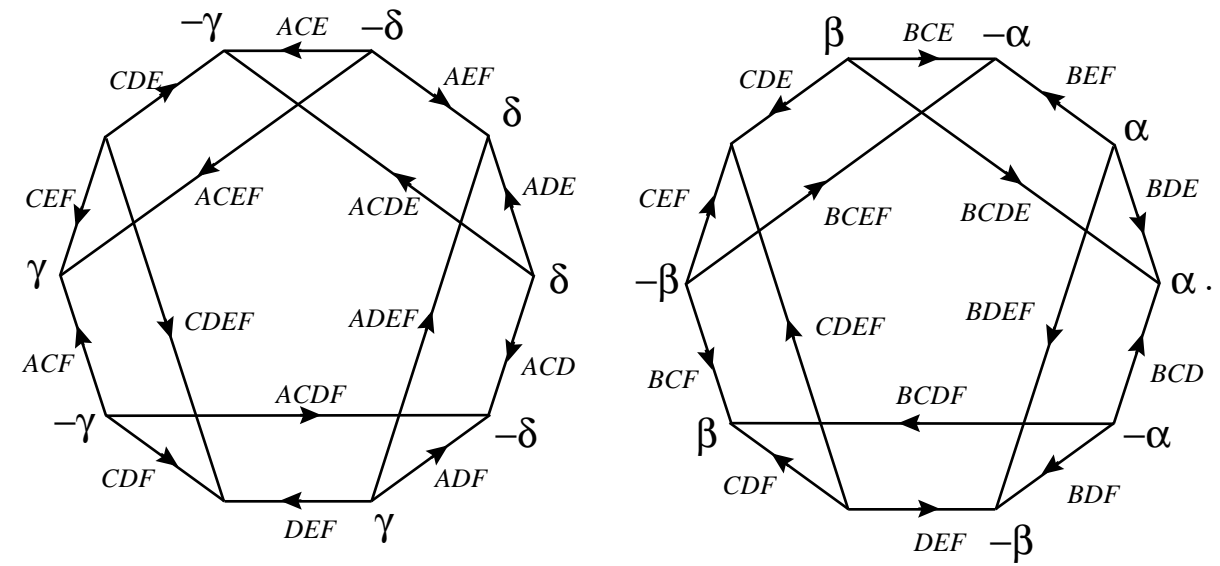
The sign configuration of the decuplet graphs before and after the 4-2 move should coincide since the overall boundary of the final two 4-simplexes have the same boundary tetrahedra as that of the initial four 4-simplexes as shown in (5.9). This requirement leads to the following relations:

$$
\alpha=\delta=-, \quad \beta=-\gamma=-\epsilon
$$

We have thus obtained several constraints from the consistency of 4-2 move.

We can use the similar arguments as 4-2 move for 5-1 and 3-3 moves to get constraints on the sign ambiguity. It turns out that 4-2 move and 5-1 move eventually give the same constraint,

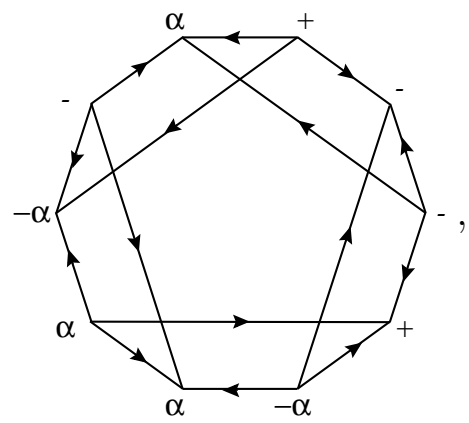

while 3-3 move give the following constraint:

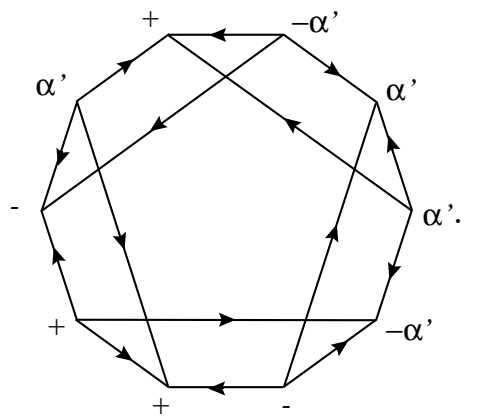

There are two consistent solutions satisfying (5.20) and (5.21)
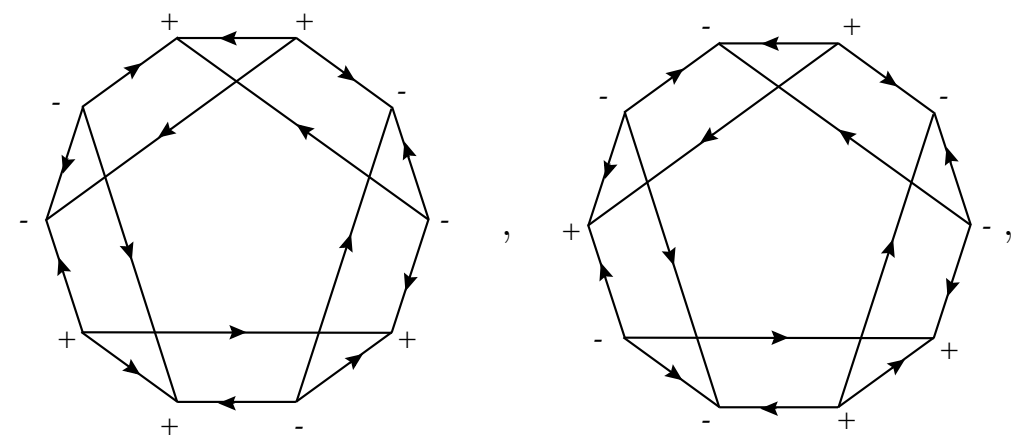

where the first solution corresponds to $\alpha=+$ and $\alpha^{\prime}=-$ while the second solution is obtained by taking $\alpha=-$ and $\alpha^{\prime}=+$ with totally reversed sign factors. At this 
stage we point out that our generalized $15-j$ symbol is different in sign factors from the one given by Crane and Yetter 16.

We have thus obtained complete expression of the generalized $15-j$ symbol with the correct sign factors. Then the full analytic expression of the Pachner move invariant partition function is

$$
Z_{L B F}=\sum_{J} \prod_{\text {site }} \Lambda^{-1} \prod_{\text {link }} \Lambda \prod_{\text {triangle }}(2 J+1) \prod_{\text {tetrahedron }}(2 J+1) \prod_{4-\text { simplex }}\left\{\begin{array}{ccccc}
J_{1} & J_{2} & J_{3} & J_{4} & J_{5} \\
J_{6} & J_{7} & J_{8} & J_{9} & J_{10} \\
J_{11} & J_{12} & J_{13} & J_{14} & J_{15}
\end{array}\right\}
$$

where we have used the first sign convention of (5.22). Here the $15-j$ symbol can be defined by

$$
\begin{aligned}
\left\{\begin{array}{ccccc}
J_{1} & J_{2} & J_{3} & J_{4} & J_{5} \\
J_{6} & J_{7} & J_{8} & J_{9} & J_{10} \\
J_{11} & J_{12} & J_{13} & J_{14} & J_{15}
\end{array}\right\} & =\sum_{\text {all } m_{i}}(-)^{\sum_{i=1}^{15}\left(J_{i}-m_{i}\right)}\left(\begin{array}{ccc}
J_{1} & J_{7} & J_{6} \\
m_{1} & m_{7} & m_{6}
\end{array}\right)\left(\begin{array}{ccc}
J_{3} & J_{8} & J_{7} \\
-m_{3} & -m_{8} & -m_{7}
\end{array}\right) \\
& \times\left(\begin{array}{ccc}
J_{4} & J_{8} & J_{9} \\
m_{4} & m_{8} & m_{9}
\end{array}\right)\left(\begin{array}{ccc}
J_{1} & J_{9} & J_{10} \\
-m_{1} & -m_{9} & -m_{10}
\end{array}\right)\left(\begin{array}{ccc}
J_{2} & J_{11} & J_{10} \\
m_{2} & m_{11} & m_{10}
\end{array}\right) \\
& \times\left(\begin{array}{ccc}
J_{4} & J_{11} & J_{12} \\
-m_{4} & -m_{11} & -m_{12}
\end{array}\right)\left(\begin{array}{ccc}
J_{5} & J_{13} & J_{14} \\
m_{5} & m_{13} & m_{14}
\end{array}\right)\left(\begin{array}{ccc}
J_{2} & J_{14} & J_{13} \\
-m_{2} & -m_{14} & -m_{13}
\end{array}\right) \\
& \times\left(\begin{array}{ccc}
J_{3} & J_{14} & J_{15} \\
m_{3} & m_{14} & m_{15}
\end{array}\right)\left(\begin{array}{ccc}
J_{5} & J_{15} & J_{6} \\
-m_{5} & -m_{15} & -m_{6}
\end{array}\right),
\end{aligned}
$$

which is graphically equivalent to

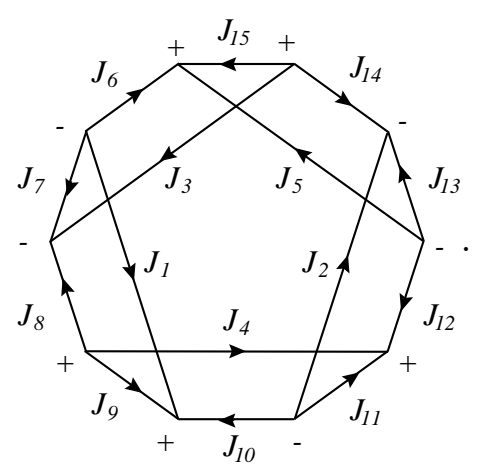

Another sign convention in (5.22) can be equally used to express the generalized 15- $j$ symbol. 


\section{Conclusion and Discussions}

We have shown that the partition function of the lattice version of $B F$ action with vanishing holonomy constraint leads to the product of $15-j$ symbols with some particular combinations of angular momentum factors and regularized constants. We have then explicitly proved by graphical method that the partition function is Pachner move invariant if we take particular choice of sign assignments for the trivalent vertices of decuplet diagrams corresponding to the 4-simplexes. This means that the partition function is independent of how the simplicial manifold is divided by 4-simplexes. This is to do with the fact that the partition function of the lattice version of $B F$ action is diffeomorphism invariant or even topological invariant in a stronger sense. Since the partition function is invariant how much finely we divide the 4-dimensional simplicial manifold in consideration, the continuum limit of the lattice $B F$ action is expected to approach naively to the continuum $B F$ action. The vanishing holonomy constraint can be then interpreted as a gauge fixing condition to align the $B^{a}$ field and the curvature $F^{a}$.

There are some desirable features in this formulation. Firstly we have found the lattice formulation of "4-dimensional gravity" which is first order formalism and is thus described by local variable $B$ and spin connection $U=e^{i \omega}$. This is the first 4-dimensional example of lattice gravity model which specifies the location of the local fields $B$ which could possibly be related to the metric and the lattice spin connection $U=e^{i \omega}$ on the 4-dimensional simplicial manifold. This lattice formulation is a beautiful unification of the idea of lattice QCD and the formulation of Regge calculus in the sense that the curvature generated by the product of link variables are concentrated on the simplexes where Regge calculus claims as the location of gravitational curvature. Secondly the magnitude of the $B$ field is naturally discretized due to the logarithmic form of our lattice $B F$ action. Thus the discreteness of the area of triangles on the original lattice is a natural consequence of the action. It should be compared with the result of Rovelli and Smolin who claimed to get the discreteness of the area of triangles in the form $\sqrt{J(J+1)}$ from the analysis of an area operator 26.

As we have already pointed out that the identification of $B=*(e \wedge e)$ renders the $B F$ action into the Palatini type of Einstein-Hilbert action. On the other hand in our $B F$ action we have taken the gauge algebra as one of the chiral counter part of 
$S O(4)$ algebra in the decomposition of the Euclidean local Lorentz group, $S O(4) \simeq$ $S U(2)_{L} \times S U(2)_{R}$. There are several possibilities how the chiral decomposition of the local Lorentz group be related to the realistic Einstein-Hilbert action 21. Here we simply comment an interesting possibility that the action $\int_{M} B_{a}^{+} F_{a}^{+}$with the gauge group as one of the chiral partner $S U(2)_{L}$ will be formulated on the original lattice as in this paper while the action $\int_{M} B_{a}^{-} F_{a}^{-}$with the other chiral partner $S U(2)_{R}$ gauge group will be formulated on the dual lattice. This situation can be symbolically be written

$$
Z_{L B F}[S O(4)]=Z_{L B F}\left[S U(2)_{L}\right] \cdot Z_{L B F}\left[S U(2)_{R}\right]
$$

In order that this theory be realistic lattice gravity model, we need some natural constraint to relate the two chiral BF models. As we have mentioned in the end of section 3, the vanishing holonomy constraint (2.12) includes 9 relations for the chiral partner of the $S O(4)$ algebra, which is just the necessary irreducible degrees of freedom for the 1-form gauge parameter. In order to count the number of the constraint, we have assumed the chiral nature even for the spacetime suffix in addition to the local Lorentz gauge suffix. In fact there are 9 other constraints in the vanishing holonomy constraint, which may correspond to the number of constraints of the spacetime suffix of the other chiral partner. It is interesting to note that the vanishing holonomy constraint (2.12) may include both constraint in one relation, which might give some clue to find a realistic lattice gravity model.

Our proposed lattice $B F$ action (2.10) can be generalized into arbitrary dimensions. In fact $I S O(3)$ Chern-Simons gravity was formulated by the 3-dimensional version of lattice $B F$ action. We claim that $D$-dimensional lattice $B F$ action has the universal structure

$$
S_{L B F}=\sum_{x} \operatorname{tr}(X(x)[\ln \underset{\tilde{l} \in \partial \tilde{P}}{\mathbb{Q}} U(\tilde{l})]),
$$

where $X(x)$ is $(D-2)$-form located on the center of $(D-2)$-simplex, $x$, in the $D$-dimensional simplicial manifold. The lattice curvature term is defined in the similar way as the 3- and 4-dimensional cases. It should be noted that the center of the dual plaquette $\tilde{P}$ coincides with the center of the simplex $x$. The vanishing holonomy constraint may have the form either the type of (2.11) or the type (2.12) depending on the suffix structure of gauge group for $X$. It is worth to recognize at 
this stage that this lattice $B F$ action is the lattice version of the leading term in the generalized Chern-Simons action in $D$ dimensions.

In our lattice gravity formulation in 3 and 4 dimensions, 1- and 2-form fields are, respectively, introduced on 1- and 2-simplexes in the simplicial manifold: $e^{a}$ and $B^{a}$ are located on the link (1-simplex) and the triangle (2-simplex) of original lattice, respectively, while $U=e^{i \omega}$ is located on the dual link (1-simplex). There is thus natural correspondence between the form degrees and the simplex numbers. The generalized gauge theory which is formulated to include all form degrees may thus very naturally be fitting to the formulation of gauge theory on the simplicial manifold.

We know that there is no dynamical graviton in the topological model while we need the graviton as a dynamical field in the realistic 4-dimensional Einstein gravity. We believe that matter fields should be introduced to accommodate the dynamical degrees of freedom and thus would change the topological nature of the model. We know by now that the ghosts introduced by the quantization will be changed into fermionic matter fields via twisting mechanism[30]. In fact we found a mechanism that $R$ symmetry of $N=2$ supersymmetry in the twist is essentially related to the Dirac-Kähler fermion formulation[31]. The Dirac-Kähler fermion needs all form degrees of freedom and can naturally be put on the simplicial manifold.

It will be thus interesting to investigate lattice gravity formulation with matter fields on the simplicial manifold. We know that weak boson gauge field and Higgs field can be very naturally accommodated into the generalized gauge theory formulation and thus this kind of formulation possibly leads to the unified model on the simplicial manifold 24].

In our formulation we have introduced cut off dependent regularized constant. It is well known by the work of Turaev and Viro that the regularization of the PonzanoRegge model can be naturally accommodated if we introduce the $q$-deformed formulation of $3-j$ symbol [9]. We didn't mention the $q$-deformed version of the present formulation in this paper although it is the main subject among mathematicians to obtain the 4-dimensional topological invariant by the $q$-deformed formulation 17. Since our $15-j$ symbol is explicitly related to the $3-j$ symbol which has the $q$ deformed counterpart, it will be straightforward to generalize our formulation into $q$-deformed version, which is expected to correspond with BF gravity with cosmo- 
logical term 10.15.

\section{Acknowledgements}

We thank L. Crane and H.B. Nielsen for useful discussions. This work is supported in part by Japanese Ministry of Education, Science, Sports and Culture under the grant number 09640330.

\section{References}

[1] F. David, Nucl. Phys. B257 (1985) 45; Nucl. Phys. B257 (1985) 543; V.A. Kazakov, I.K. Kostov and A.A. Migdal, Phys. Lett. 157B (1985) 295; D.V. Boulatov, V.A. Kazakov, I.K. Kostov and A.A. Migdal, Nucl. Phys. B275 (1986) 641; J. Ambjørn, B. Durhuus and J. Fröhlich, Nucl. Phys. B257 (1985) 433.

[2] V.G. Knizhnik, A.M. Polyakov and A.A. Zamolodchickov, Mod. Phys. Lett. A3 (1988) 819; J. Distler and H. Kawai, Nucl. Phys. B321 (1989) 509; F. David, Mod. Phys. Lett. A3 (1988) 651.

[3] N. Kawamoto, V.A. Kazakov, Y. Saeki and Y. Watabiki, Phys. Rev. Lett. 68 (1992) 2113; Nucl. Phys. B26 (Proc. Suppl.) (1992) 584.

[4] J. Ambjørn, K.N. Anagnostopoulos, T. Ichihara, L. Jensen, N. Kawamoto, Y. Watabiki and K. Yotsuji, Phys. Lett. B397 (1997) 177; Nucl. Phys. B511 (1998) 673.

[5] H. Kawai, N. Kawamoto, T. Mogami and Y. Watabiki, Phys. Lett. B306 (1993) 19.

[6] M.E. Agishtein and A.A. Migdal, Int. J. Mod. Phys. C1 (1990) 165; Nucl. Phys. B350 (1991) 690.

[7] E. Witten, Nucl. Phys. B311 (1988/89) 46.

[8] G. Ponzano and T. Regge, in Spectroscopic and group theoretical methods in physics, ed. F.Block (North-Holland, Amesterdam, 1968). 
[9] V.G. Turaev and O.Y. Viro, Topology 31 (1992) 865;

V.G. Turaev, C. R. Acad. Sci. Paris 313 (1991) 395; J. Diff. Geom. 36 (1992) 35.

[10] H. Ooguri and N. Sasakura, Mod. Phys. Lett. A6 (1991) 3591;

H. Ooguri, Nucl. Phys. B382 (1992) 276;

N. Sasakura, Prog. Theor. Phys. Suppl. 110 (1992) 191.

[11] D. Boulatov, Mod. Phys. Lett. A7 (1992) 1629; R. Anishetty, S. Cheluvaraja, H.S. Sharatchandra and M. Mathur, Phys. Lett. B314 (1993) 387; S. Chung, M. Fukuma and A. Shapere, Int. Jour. Mod. Phys. A9 (1994) 1305; F. Archer and R.M. Williams, Phys. Lett. B273 (1991) 438; S. Mizoguchi and T. Tada, Phys. Rev. Lett. 68 (1992) 1795; B. Durhuus, H.P. Jakobsen and R. Nest, Nucl. Phys. B25 A (Proc. Suppl.) (1992) 109; F.J. Archer, Phys. Lett. B295 (1992) 199; J. Iwasaki, Jour. Math. Phys. 36 (1995) 6288; L. Freidel and K. Krasnov, Class. Quant. Grav. 16 (1999) 351; A. Cattaneo, P. Cotta-Ramusino, J. Fröhlich and M. Martellini, Jour. Math Phys. 36 (1995) 6137.

[12] N. Kawamoto, H.B. Nielsen and N. Sato, Nucl. Phys. B555 (1999) 629.

[13] A.S. Schwarz, Lett. Math. Phys. 2 (1978) 247; G. Horowitz, Comm. Math. Phys. 125 (1989) 417; D. Birmingham, M. Blau, M. Rakowski and G. Thompson, Phys. Rep. 209 (1991) 129; M. Blau and G. Thompson, Ann. Phys. 205 (1991) 130 .

[14] H. Ooguri, Mod. Phys. Lett. A7 (1992) 2799.

[15] J.C. Baez, Strings, loops, knots and gauge fields, in Knots and Quantum Gravity, ed. J. Baez (Oxford U.Press, 1994); Lett. Math. Phys. 38 (1996) 129; Class. Quant. Grav. 15 (1998) 1827; gr-qc/990587;

M. Reisenberger and C. Rovelli, Phys. Rev. D56 (1997) 3490;

L. Freidel and K. Krasnov, Adv. Theor. Phys. 2 (1998) 1221.

[16] L. Crane and D. Yetter, A categorical construction of 4d TQFTs, in Quantum Topology, eds. L. Kauffman and R. Baadhio (World Scientific, Singapore, 1993) pp.120. 
[17] L. Crane, L. Kauffman and D. Yetter, J. Knot Theory \& Ramifiations 6 (1997) 177 ;

J. Roberts, Topology 34 (1995) 771.

[18] T. Regge, Nuovo Cimento 19 (1961) 558.

[19] N. Kawamoto and H.B. Nielsen, Phys. Rev. D43 (1991) 1150.

[20] M. Caselle, A. D'adda, and L. Magnea, Phys. Lett. B232 (1989) 457.

[21] J.F. Plebanski, J. Math. Phys. 18 (1977) 2511; A. Ashtekar, Phys. Rev. Lett. 57 (1986) 2244; Phys. Rev. D36 (1987) 1587; R. Capovilla, J. Dell, T. Jacobson and L. Mason, Phys. Rev. Lett. 63 (1989) 2325; Class. Quantum Grav.8 (1991) 41.

[22] N. Kawamoto and Y. Watabiki, Commun. Math. Phys. 144 (1992) 641; Mod. Phys. Lett. A7 (1992) 1137; Commun. Math. Phys. 148 (1992) 169.

[23] N. Kawamoto and Y. Watabiki, Phys. Rev. D45 (1992) 605; Nucl. Phys. B396 (1993) 326.

[24] N. Kawamoto, Prog. Theor. Phys. Suppl. No. 134 (1999) 84.

[25] M. Kalb and P. Ramond, Phys. Rev. D9 (1974) 2273;

Y. Nambu, Phys. Rep. 23C (1976) 250.

[26] C. Rovelli and L. Smolin, Nucl. Phys. B442 (1995) 593; Erratum, ibid. B456 (1995) 753.

[27] D.A. Varshalovich, A.N. Moskalev, and V.K. Khersonskii, Quantum Theory of Angular Momentum (World Scientific, 1988).

[28] J. Ambjørn, M. Carfora, A. Marzuoli, The Geometry of Dynamical Triangulations (Springer-Verlag, 1997).

[29] N.H. Christ, R. Friedberg, T.D. Lee, Nucl. Phys. B202 (1982) 89.

[30] E. Witten, Commun. Math. Phys. 117 (1988) 353; Int. J. Mod. Phys. A6 (1991) 2775; Nucl. Phys. B340 (1990) 281; J.Math.Phys. 35 (1994) 5101.

[31] N. Kawamoto and T. Tsukioka, hep-th/9905222. 